\title{
A Novel Network Science and Similarity Searching-Based Ap- proach for Discovering Potential Tumor Homing Peptides from Antimicrobials
}

\author{
Maylin Romero 1, Yovani Marrero-Ponce 2,*, Hortensia Rodríguez ${ }^{1}$, Guillermin Agüero-Chapin, ${ }^{3,4}$ Agostinho An- \\ tunes, ${ }^{3,4}$ Longendri Aguilera-Mendoza ${ }^{5}$, and Felix Martinez-Rios ${ }^{6}$.
}

1 School of Chemical Sciences and Engineering, Yachay Tech University, Hda. San José s/n y Proyecto Yachay, Urcuquí 100119, Ecuador; hmrodriguez@yachaytech.edu.ec (https://orcid.org/0000-0001-6910-5685) and maylin.romeroh@gmail.com (http://orcid.org/0000-0003-1031-5308)

2 Universidad San Francisco de Quito (USFQ), Grupo de Medicina Molecular y Traslacional (MeM\&T), Colegio de Ciencias de la Salud (COCSA), Escuela de Medicina, Edificio de Especialidades Médicas; and Instituto de Simulación Computacional (ISC-USFQ), Diego de Robles y vía Interoceánica, Quito, 170157, Pichincha, Ecuador; ymarrero@usfq.edu.ec; http://www.orcid.org/0000-0003-2721-1142

3 CIIMAR/CIMAR, Centro Interdisciplinar de Investigação Marinha e Ambiental, Universidade do Porto, Terminal de Cruzeiros do Porto de Leixões, Av. General Norton de Matos, s/n, 4450-208 Porto, Portugal; gchapin@ciimar.up.pt (https://orcid.org/0000-0002-9908-2418) and aantunes@ciimar.up.pt (http://orcid.org/0000-0002-1328-1732)

4 Departamento de Biologia, Faculdade de Ciências, Universidade do Porto, Rua do Campo Alegre, 4169-007 Porto, Portugal.

5 Departamento de Ciencias de la Computación, Centro de Investigación Científica y de Educación Superior de Ensenada (CICESE), Baja California 22860, Mexico. longendri@gmail.com; https://orcid.org/0000-0002$\underline{2421-0382}$

6 Facultad de Ingeniería, Universidad Panamericana, Augusto Rodin No. 498, Insurgentes Mixcoac, Benito Juárez, 03920 Ciudad de México, CDMX, México. felix.martinez@up.edu.mx; http://orcid.org/0000-00022346-5922.

* Correspondence: ymarrero@usfq.edu.ec or ymarrero77@yahoo.es; Tel.: +593-2-297-1700 (ext. 4021). http://www.uv.es/yoma/ or http://ymponce.googlepages.com/home; ORCID ID: http://www.orcid.org/0000$\underline{0003-2721-1142}$

\begin{abstract}
Peptide-based drugs are promising anticancer candidates due to their biocompatibility, and low toxicity. Particularly, tumor homing peptides (THPs) have the ability to bind specifically to cancer cells receptors and tumor vasculature. Despite their potential to develop antitumor drugs, there are few available prediction tools to assist the discovery of new THPs. Two webservers based on machine learning models are currently active, the TumorHPD (https://webs.iiitd.edu.in/raghava/tumorhpd) and the THPep (http://codes.bio/thpep), and more recently the SCMTHP (SCMTHP (pmlabstack.pythonanywhere.com), based on scoring card method. Herein, a novel method based on network science and similarity searching implemented in the starPep toolbox (http://mobiosd-hub.com/starpep/) is presented for THPs discovery. The approach leverages from exploring the structural space of THPs with Chemical Space Networks (CSNs) and from applying centrality measures to identify the most relevant and non-redundant THPs sequences within the CSN. Such THPs were considered as queries (Qs) for multi-query similarity searches that applies a group fusion (MAX-SIM rule) model. The resulting multi-query similarity searching models (SSMs) were validated with three benchmarking datasets of THPs/non-THPs. Predictions achieved accuracies ranged from 92.64 to $99.18 \%$ and Matthews Correlation Coefficients between 0.894-0.98, outperforming state-of-the-art predictors. The best model was applied to repurpose AMPs from the starPep database as THPs, which were subsequently optimized for the TH activity. Finally, 54 promising THP leads were discovered, and their sequences were analyzed to encounter novel motifs. These results demonstrate the potential of CSNs and multi-query similarity searching for a rapid and accurate identification of THPs.
\end{abstract}

Keywords: Cancer, tumor homing peptide, in silico drug discovery, complex network, chemical space network, centrality measure, similarity searching, group fusion, motif discovery, starPep toolbox software. 


\section{Introduction}

Cancer is a group of diseases developed in different cell and tissue types, and corresponds to the second leading cause of death globally [1]. It is based on the abnormal growth of cells due to an inherited genetic mutation or induced by the environment [2]. Despite novel therapies development for cancer treatment, improving chemotherapeutic drugs' specificity towards cancer cells remains a challenge [2,3]. Additionally, cancer cells are generating multidrug resistance (MDR) [4]. Consequently, in the pharmaceutical industry, there is a need to develop new anticancer agents with a different mode of action to tackle the current drug resistance of cancer cells without being cytotoxic to healthy ones [2]. To fill this gap, peptides have emerged as a potential therapeutic alternative against cancer. From 2015 to 2019, 15 peptides or peptide-containing molecules were approved by the FDA as drugs demonstrating the growing interest of the scientific community [5].

Peptides have different biochemical and therapeutic properties than small molecules and proteins making them attractive to the pharmaceutical and biotechnological industry $[6,7]$. At being smaller than proteins allows peptides to penetrate tissues more easily, have low cost, more accessible synthesis, and do not require folding to be biologically active [8]. In contrast to small molecules, they have higher specificity and efficacy due to representing the smallest functional part of a protein [9]. Moreover, they are not supposed to interact with the immune system, are biocompatible, have tunable bioactivity, and have low cytotoxicity due to the degradation products are amino acids [10-14]. Hence, peptidebased drugs open a new door to an improved cancer diagnosis and treatment.

Tumor blood and lymphatic vasculature differ molecularly and morphologically from normal lymphatic and blood vessels [15]. Tumor homing peptides (THPs) take advantage of this peculiarity. Thus, they are widely investigated as drug carriers and for imaging purposes on oncology treatments and diagnosis [16]. First-generation of THPs have RGD (Arg-Gly-Asp) and NGR (Asn-Gly-Arg) motifs. RGD peptides have the characteristic of selectively binding to $\alpha$ integrins expressed in vascular endothelial cells of the tumor and metastatic tumor cells, while NGR to aminopeptidase N (APN) receptors $[17,18]$. Although, there are non-RGD neither NGR peptides that home tumor blood vasculature and cancer cells by interactions with other receptors, such as endothelial growth factor receptor (EGFR) [19-23].

THPs are discovered by using in vitro and ex vivo/in vivo phage display technology, which is time-consuming, expensive and may not translate to humans due to differences between the animal models and humans [24-26]. For these reasons, bioinformatics tools such as databases and webservers are being employed for the accurate prediction of novel THPs [26-28]. In this way, short sets of the most promising THPs become the candidates for posterior experimental verification.

To date, the databases available for experimentally validated THPs are TumorHoPe (included 744 THPs) [27] and starPepDB (included 659 THPs) [29], and the available TH activity predictors are TumorHPD [26], THPep [28], and SCMTHP [30]. TumorHPD uses the supervised ML method Support Vector Machine (SVM) as a classifier with three features, amino acid composition, dipeptide composition, and binary profile patterns, achieving $86.56 \%$ as the highest accuracy [26]. The second ML method, THPep, has a Random Forest (RF) classifier with three features, amino acid composition, dipeptide composition, and pseudo amino acid composition, resulting in $90.13 \%$ of maximum overall accuracy [28]. However, the datasets used for training and testing both ML models contain peptides with high similarity sequences. On the other hand, SCMTHP is the most recently reported method based on the scoring card method (SCM) [30]. It determines the propensity scores for the amino acids and dipeptides composition accounting for THP sequences and applies a threshold value to discriminate between THP and non-THPs. Nonetheless, the performance of SCMTHP is similar to ML-based predictors, achieving a maximum accuracy of $82.7 \%$.

Recently, Marrero-Ponce et al. published a new software named starPep toolbox, which is aimed to perform network analyses on the integrated graph database called starPepDB, which include the most comprehensive and non-redundant database of antimicrobial peptides (AMPs) [29,31]. We propose an alternative methodology to identify 
potential THPs by combining of network science with multi-query similarity searching against the AMPs of starPepDB. We used the starPep toolbox software as the main bioinformatics tool and the Chemical Space Network (CSN) to represent the chemical space of peptides as a coordinate-free system. To the best of our knowledge, there are no reported studies where data mining and screening is supported by network science to discover peptides for pharmaceutical purposes [29]. Firstly, we built models of representative and non-redundant THPs using centrality analysis and a supervised retrospective similarity searching to perform the TH activity prediction. The outstanding model, named THP1, predicted the TH activity of three benchmarking datasets of THPs/non-THPs achieving accuracies between 92.64-99.18\% and Matthews Correlation Coefficient (MCC) between 0.894-0.98, demonstrating the feasibility of this new methodology. Then, we performed a hierarchical screening for drug repurposing using network-based algorithms implemented in the starPep toolbox, the best model THP1, local alignments, and webservers to predict relevant activities related to the TH. Their TH activity was optimized by generating random libraries, where the peptide undergoes amino acid's stochastic substitutions at different positions. Finally, a set of 54 potential THPs from AMPs is proposed, where common motifs were identified.

\section{Materials and Methods}

The overall workflow of this report, shown in Figure 1, is based on two steps: i) generation/selection of the model of representative THPs from starPepDB in starPep toolbox, and ii) prediction of potential new THPs. In the first step, some models of representative THPs from starPepDB were built using different centrality measures to rank the nodes and extract the representative and less similar sequences by applying local alignment. Then, the best multi-query similarity searching model (SSM) was selected by the classification performance and its ability to retrieve correctly THPs from benchmark THPs databases by using group fusion (MAX-SIM rule) similarity searching.
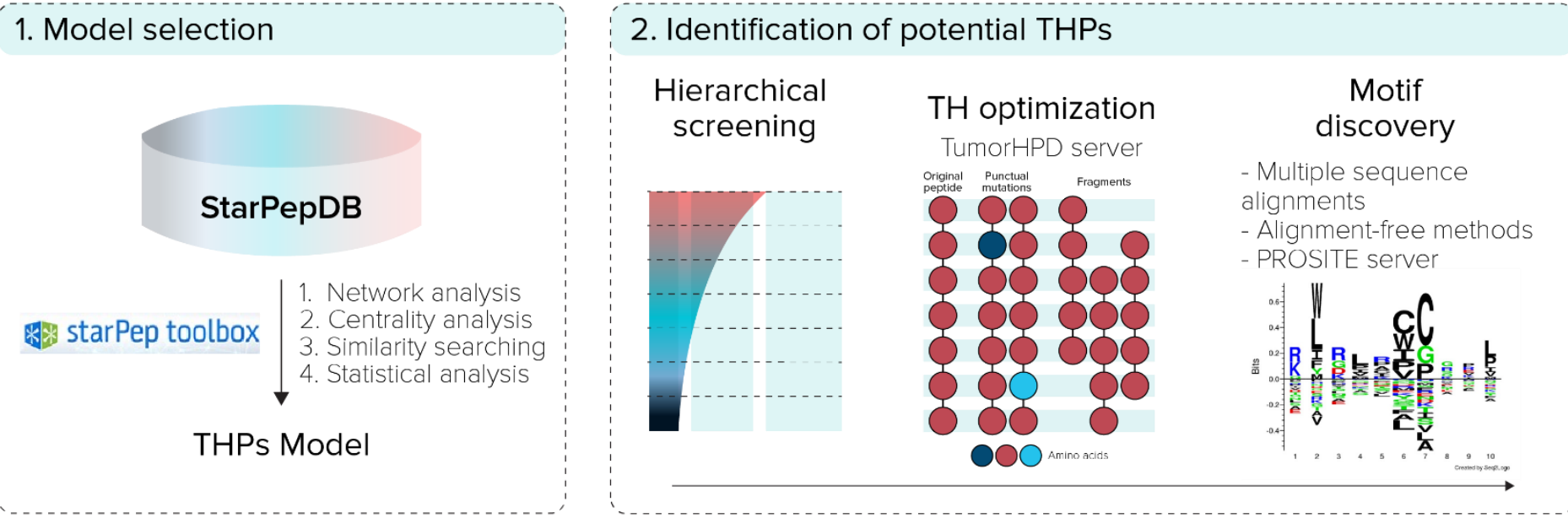

Figure 1. General overview of the experimental procedure.

In the second step, the model was used to perform similarity searching to repurpose AMPs as THPs from starPepDB, and their TH activity was optimized using the TumorHPD server. Additionally, sequence motifs were found from the set of potential THPs using multiple sequence alignments [3235], alignment-free methods [36], and PROSITE server (https://www.genome.jp/tools/motif).

\subsection{StarPep Toolbox Software}

The starPep toolbox uses FASTA files as inputs, and includes the starPepDB. Peptides are represented as nodes connected by an edge if they have any relationship. It can perform querying, filtering, visualization of networks, scaffold extractions, single or multiple queries similarity searching, and analysis of peptides by graph networks [29,31].

Networks can be built based on the metadata of peptides or based on the pairwise similarity measures calculated for their respective sequence. In metadata networks, nodes 
are connected by a specific parameter in common, such as origin, the target which assessed against, functionality, the database where they come from, the cross-reference, $\mathrm{N}$ terminus, C-terminus, or amino acid composition. In similarity networks, peptides are codified by descriptors, such as length, net charge, isoelectric point, molecular weight, Boman index, indices based on aggregation operators, hydrophobic moment, average hydrophilicity, hydrophobic periodicity, aliphatic index, and instability index $[29,31,37]$. Moreover, networks are visualized using different layouts, such as Fruchterman Reingold [38].

Networks can be clustered, and communities are optimized using the Louvain method [39]. Moreover, the centrality of each node can be particularly measured by harmonic, community hub-bridge, betweenness, and weighted degree. Centrality is crucial to perform scaffold extractions because peptides are ranked according to their centrality score, and then redundant sequences are removed, prioritizing the most central. Thus, scaffold extractions depend on the type of centrality applied.

On the other hand, similarity searching, which is the basis of this study, is performed using a set of queries against a target dataset, where different percentages of identity (or similarity thresholds) can be applied. An identity score is a number between $0-1$, and it is calculated using the Smith-Waterman local alignment with BLOSUM 62 substitution matrix [40]. Multiple queries similarity searching works using the group fusion model explained in the following section.

\subsection{Model Selection}

The dataset of reported THPs was extracted from starPepDB in the starPep toolbox. All 45120 peptides contained in starPepDB were filtered by the "Tumor Homing" query in the metadata function, where 659 entries were obtained (SI1-A).

\subsubsection{Network Analysis}

\subsubsection{Similarity Threshold Analysis}

Network analysis of peptides was performed by building the CSN of 659 THPs in the the starPep toolbox. To choose the appropriate similarity threshold to build the network of THPs, CSNs were built by varying in 0.05 the cut-off value from 0.10 to 0.90 ( 17 CSNs in total). Some metrics were retrieved from each CSN using the starPep toolbox, such as density, number of communities, modularity, and number of singletons.

By default, when CSN is built, nodes with higher than $98 \%$ of similarity were removed using the local alignment Smith-Waterman algorithm. The similarity metric used to establish the pairwise similarity relationships between nodes was the min-max normalized Euclidean distance. Then, a centrality was calculated and those nodes with 0 as vertex degree identified as outliers and then removed, remaining the giant (or connected) components of the CSN, i.e., subgraph where all nodes are connected. In this case, community hub-bridge centrality was calculated. However, any centrality measure could have been calculated since singletons always have zero centrality. After that, the network was clustered and the modularity optimized using the Modularity optimization algorithm based on the Louvain method [39].

The network was saved as a Graph ML file to be opened in Gephi [41] for subsequent calculation of ACC. Finally, density, modularity, and ACC as a function of similarity threshold were graphed in Origin to decide what similarity threshold is the best.

\subsubsection{Network Characterization}

CSN of the giant components derived from the application of the best similarity threshold is characterized by the number of nodes, edges, outliers, density, number of communities, and modularity. These parameters were obtained from starPep toolbox while ACC, diameter (larger shortest path), average path length, and a total of triangles were drawn from Gephi; and the distribution degree. These parameters allow knowing the topology, and structural patterns of the CSN.

For network visualization, Force Atlas 2 was used as a layout algorithm where colors represent different clusters, and node size means how central is the node according to the 
community hub-bridge centrality. Network visualization aims to obtain an aesthetically pleasing and understandable graph where nodes are not overlapped.

On the other hand, CSN of outliers was built with a cut-off of 0.30 to procure an appropriate density and, then, it was clustered. Moreover, a subsequent scaffold extraction was applied based on hub-bridge centrality, and on 30\% identity from local alignment.

The network of outliers was characterized according to the number of nodes, edges, and communities, density, modularity, average degree, ACC, and diameter obtained before scaffold extraction, and the number of nodes and edges obtained after scaffold extraction. For network visualization, Fruchterman Reingold was used as a layout algorithm, colors represent different clusters while node size displays how central it is according to hub-bridge measure.

\subsubsection{Centrality Analysis}

The most influential nodes were used to find the new potential THPs, and centrality is the crucial parameter that provides this information. Thus, the four available centrality types in the starPep toolbox, weighted degree, community hub-bridge, betweenness, and harmonic, were calculated and normalized using the min-max method. Then, redundant peptides were removed by applying the scaffold extraction procedure that is described as follows: peptides were ranked based on the scores obtained after centrality calculation and used $30 \%$ similarity cut-off of local identity from the Smith-Waterman algorithm to retrieve sets of sequences with a maximum of $30 \%$ similarity [40]. Subsequently, nodes with $10 \%$ lower centrality than the most central node were removed in each metric. The sets obtained after applying this process were named as $30+10 \%$.

On the other hand, harmonic and weighted degree were calculated, normalized, and redundant peptides were removed by applying the scaffold extraction procedure using four different similarity cut-offs of local identity, 30, 40, 50, and $60 \%$.

\subsubsection{Similarity Searching Model for THPs Prediction}

This study's proposed method for discovering potential THPs was based on similarity searching. For that reason, multiple query similarity searching models (SSMs) composed of several queries representing the most important and less redundant nodes of CSN and a similarity threshold were tested against datasets that contain well-known THPs/non-THPs through similarity searching. The recoveries from the similarity searching were statistically evaluated to select the best model for identifying potential THPs within the AMPs.

\subsubsection{Query Datasets (Reference Sequences)}

The retrieved sets after applying scaffold extractions at each centrality measure, the two sets of outliers, combinations of outliers with sets obtained from centrality-based scaffold extractions, and combinations between sets obtained from scaffold extractions performed using different centrality metrics were used as queries (Qs). In total, we tested 22 sets of Qs, where twelve sets resulted from the application of the scaffold extraction procedures, two sets of outliers, and eight sets resulted from the combination between sets.

\subsubsection{Target Databases}

Three training datasets that consider well-known THPs and randomly generated non-THPs [42] were used as the target or calibration for the recovery. THPep, TumorHPD and SCMTHP employed these datasets for training their methods [26,30,42].

- Main dataset: 651 experimentally validated THPs and 651 random non-THPs (SI1-B). They were collected from TumorHoPe [27], and the literature [26].

- Small dataset: 469 experimentally validated THPs and 469 random non-THPs (SI1C). They are peptides derived from the Main dataset with 4-to-10 aa residues.

- Main90 dataset: 176 THPs and 443 non-THPs (SI1-D). They are peptides from the Main dataset with equal or lower than $90 \%$ of sequence similarity.

Main and Small datasets were retrieved from Ref. [26], while Main90 from Ref. [27].

2.2.3.3. Group fusion 
Group fusion is based on the variation of the query (reference peptide), but keeping constant the identity measure [43]. Each peptide's identity score is calculated from the target dataset varying the Qs. The fusion group's algorithm associates a fused score to each target peptide, i.e., the maximum similarity (MAX-SIM) scores from all resulting identity scores against the Qs. Therefore, considering peptide $S$ from the target dataset, reference peptide $Q$ from the $Q s$, and the identity score $I(S, Q)$ the MAX-SIM score obtained, the algorithm assigns $I(S, Q)$ as the fused score to peptide $S$. The local identities were calculated with the Smith-Waterman, and is a number between 0-1, being one the maximum identity. The procedure is illustrated in Figure 2.

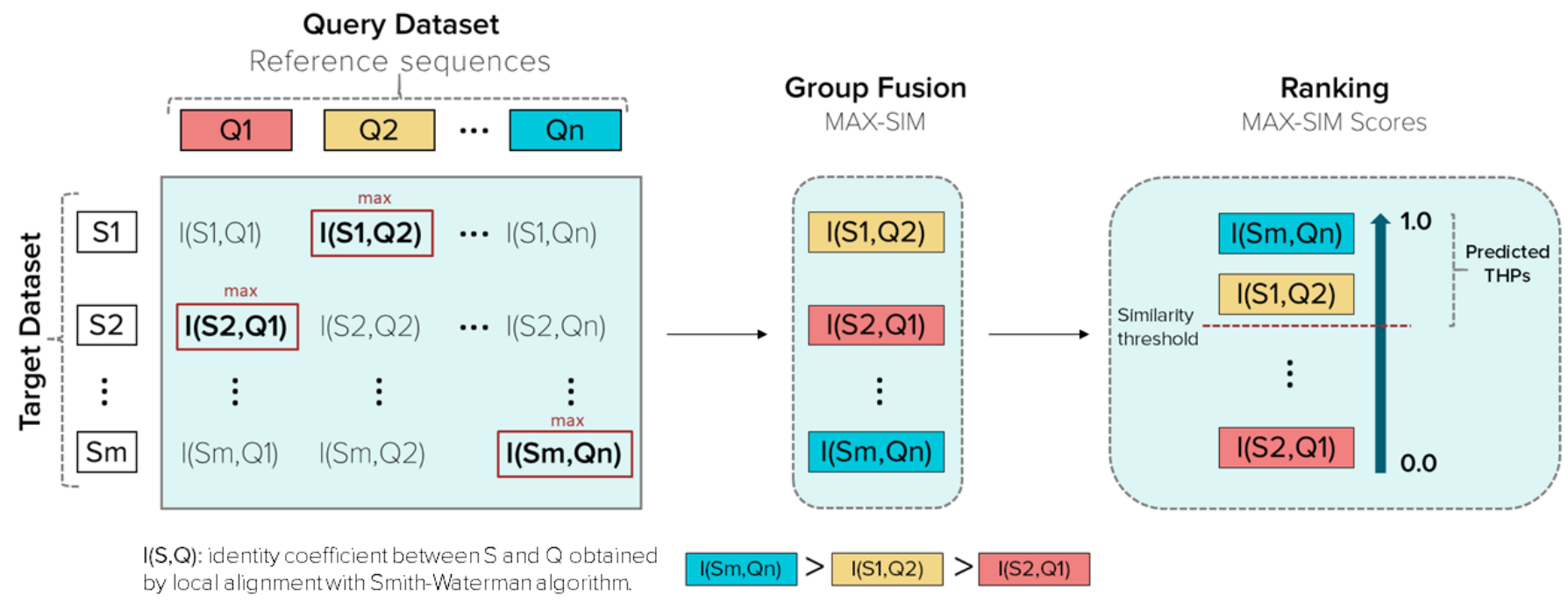

Figure 2. Schematic representation of the group fusion and similarity searching processes. $Q$ is a peptide from a query dataset, $\mathrm{n}$ the number of peptides contained in a query dataset, $\mathrm{S}$ is a peptide from the target dataset (Main, Small or Main90 dataset), $\mathrm{m}$ is the number of peptides included in the target dataset (1302, 938 or 619 , respectively). The similarity threshold is related to the percentage of identity.

\subsubsection{Retrospective Similarity Searching.}

Main Dataset was imported to starPep toolbox. The similarity searching was performed using the "Multiple query sequences" option of the software and the Qs obtained from $30+10 \%$ similarity cut-offs of local alignment and outliers. The group fusion is applied by default during the similarity searching, and results were ranked according to the fused score (MAX-SIM value). Subsequently, seven different percentages of identity (similarity thresholds), 30, 40, 50, 60, 70, 80, and 90\%, were tested, where peptides with identities equal or higher than the applied threshold were retrieved as predicted THPs. Figure 2 illustrates how the similarity searching works.

The rescued nodes, i.e., predicted THPs, were statistically evaluated to validate the prediction. Thus, it is possible to identify the two centrality measures and percentages of sequence identity with the best performance.

Then, similarity searching was performed using only the sets of the best two centrality measures as Qs: harmonic and weighted degree, and 30, 40, 50, 60, and 70\% of identity. In Small and Main90 datasets, only the sets of harmonic and weighted degrees were used as Qs, applying 40,50 , and $60 \%$ of identity for recovery. In total 98 different SSMs were evaluated.

\subsubsection{Statistical Analysis}

The ability of the SSMs to predict THPs was validated by the measurement of their accuracy $(A c)$, kappa $(\kappa)$, sensitivity $(\mathrm{Sn})$, specificity $(\mathrm{Sp})$, the precision of positives and negatives ( $\mathrm{P}_{\mathrm{pos}}$ and $\mathrm{P}_{\text {neg, }}$ respectively), MCC, and false accept rate (FAR\%) using the following formulas.

$$
\mathrm{AC}=\frac{\mathrm{TP}+\mathrm{TN}}{\mathrm{TP}+\mathrm{TN}+\mathrm{FP}+\mathrm{FN}}
$$




$$
\begin{aligned}
& \kappa=\frac{\mathrm{Po}-\mathrm{Pc}}{1-\mathrm{Pc}}, \\
& \mathrm{Sn}=\frac{\mathrm{TP}}{\mathrm{TP}+\mathrm{FN}}, \\
& \mathrm{Sp}=\frac{\mathrm{TN}}{\mathrm{TN}+\mathrm{FP}^{\prime}}, \\
& \mathrm{P}_{\mathrm{pos}}=\frac{\mathrm{TP}}{\mathrm{TP}+\mathrm{FP}}, \\
& \mathrm{P} \text { neg }=\frac{\mathrm{TN}}{\mathrm{TN}+\mathrm{FN}}, \\
& \mathrm{MCC}=\frac{\mathrm{TP} \times \mathrm{TN}-\mathrm{FP} \times \mathrm{FN}}{\sqrt{(\mathrm{TP}+\mathrm{FP}) \times(\mathrm{TP}+\mathrm{FN}) \times(\mathrm{TN}+\mathrm{FP}) \times(\mathrm{TN}+\mathrm{FN})}} \\
& \mathrm{FAR} \%=\frac{\mathrm{FP}}{\mathrm{FP}+\mathrm{TN}} \times 100,
\end{aligned}
$$

where, TP is the number of true positives, TN is the number of true negatives, FP is the number of false positives, FN is the number of false negatives, Po is the relative observed agreement between the observers equal to the $A c$, and $P_{c}$ is the expected chance agreement calculated by the formula $P C=\frac{(\mathrm{TP}+\mathrm{FP}) \times(\mathrm{TP}+\mathrm{FN})+(\mathrm{FN}+\mathrm{TN}) \times(\mathrm{FP}+\mathrm{TN})}{(\mathrm{TP}+\mathrm{TN}+\mathrm{FP}+\mathrm{FN})^{2}}$.

Finally, the best 9 SSMs were compared and ranked using the Friedman test-based analysis performed in KEEL [44], open-source software from Java. The Friedman test identified the best model based on the statistical metrics previously shown [45]. Moreover, it allows us to compare the models and determine if their difference is statistically significant and not due to chance. The confusion or classification matrix of the best model was constructed. The best models were compared with reported ML models used for THPs prediction, TumorHPD and THPep, using the same three calibration datasets.

\subsection{Identification of Potential THPS}

\subsubsection{Hierarchical Screening}

Drug repurposing is an alternative methodology widely applied to discover drugs because it reduces approval time for their clinical use [46,47]. Thus, firstly, we repurposed AMPs from starPepDB as THPs.

1. Pipeline Prospective Screening. First, AMPs without reported TH activity and toxicity with a sequence length between 3 to 25 residues were filtered from the chemical space of starPepDB. Secondly, the "Scaffold extraction" option removed AMPs with higher than $95 \%$ sequence similarity by local alignment. Thirdly, multiple query similarity searching was performed using the best SSM (THP1), obtained in the previous section, to explore the chemical space of non-THPs, non-toxic, and non-redundant peptides with a length of 3-25 aa, using $60 \%$ as similarity threshold. In the recovered set, peptides with a similarity score of 1 were removed.

2. Activity Prediction. Peptides with reported tumor homing activity in the literature were removed since the main objective of this study is to identify novel THPs. Then, theoretical activities of virtual hits were predicted using webservers TumorHPD [26], THPep [28], AntiCP [48], CellPPD [49], ToxinPred [50], and HemoPI [51], to corroborate their potential as THPs and prioritize those that do not harm healthy cells. The activities of interest were tumor homing, anticancer, cell-penetrating, toxicity, and hemolysis. The SVM thresholds used were 0.30 in servers TumorHPD, AntiCP, and CellPPD, and 0 in server ToxinPred.

3. Redundancy Reduction by Network Analysis. CSN of hits was built, clustered, and the modularity was optimized using the Louvain method in the starPep toolbox. Then, harmonic and weighted degree centralities were calculated to perform a scaffold extraction using a $60 \%$ identity as the threshold.

4. Visual Mining. The neighborhood of well-known THPs of each potential THPs was visualized using the starPep toolbox. CSN of 659 THPs in starPepDB was built using 0.60 as cut-off, clustered, and optimized modularity. Hits obtained in the previous 
step after scaffold extraction were embedded into the CSN of 659 THPs to study the neighborhood of each peptide. Hence, the 3-nearest neighbors from 659 THPs directly attached to each hit, were visualized. If two peptides shared the same 2 or 3nearest neighbors, one of them was prioritized, choosing the one with better-predicted activities.

\subsubsection{Tumor Homing Activity Optimization}

Lead hits detected from hierarchical virtual screening are AMPs from starPepDB with a natural or designed activity different from tumor homing. That's the reason why their tumor homing action should be enhanced. Lead hits were optimized by punctual amino acid mutations using the "Designing of Tumor Homing Peptides" module of TumorHPD (https://webs.iiitd.edu.in/raghava/tumorhpd/peptide.php), and the procedure is shown in Figure 3. Both, lead and mutated sequences were shortened into fragments 5, 10 , and 15 residues in length using the same server.

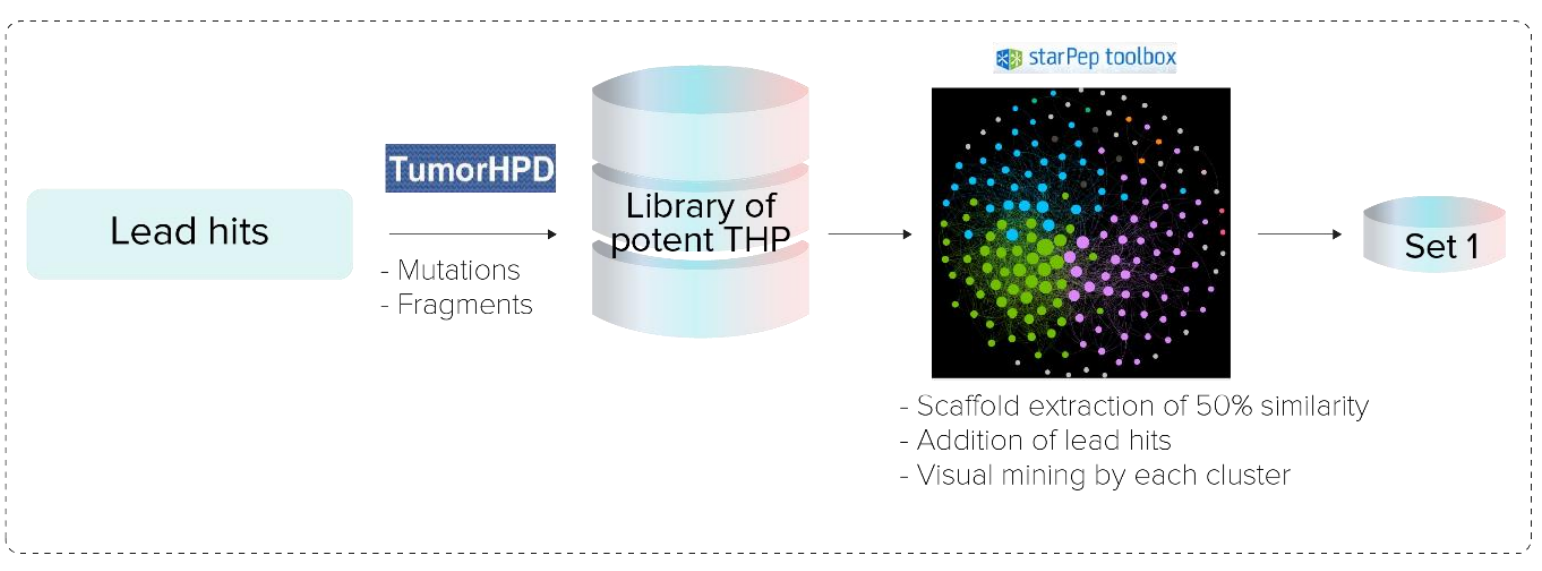

Figure 3. Procedure to optimize tumor homing activity of lead hits.

The optimized sequences showing a higher tumor homing activity score than parent hits were analyzed by CSN in the starPep toolbox using 0.60 as the similarity threshold to build the network. Besides, tumor homing, toxicity, hemolytic, anticancer, and cell-penetrability were predicted using servers listed below THPep (http://codes.bio/thpep), TumorHPD (https://webs.iiitd.edu.in/raghava/tumorhpd), AntiCP (https://webs.iiitd.edu.in/raghava/anticp2), CellPPD (https://webs.iiitd.edu.in/raghava/cppsite1), ToxinPred https://webs.iiitd.edu.in/raghava/toxinpred, and HemoPI https://webs.iiitd.edu.in/raghava/hemopi. Redundant sequences with higher than 50\% of similarity were removed by scaffold extraction.

The optimized sequences and parent hits were merged, and its corresponding CSN was built using 0.50 of cut-off and clustered. Next, harmonic centrality was calculated. Each cluster was analyzed separately to prioritize the most central, potent, non-toxic, and non-hemolytic lead THPs. Finally, the heat map and histogram of pairwise sequence identity of lead compounds were constructed to explore their structural diversity.

\subsubsection{Motif Discovery}

\subsubsection{Multiple Sequence Alignments}

As the resulting potential THPs were hard-to-align sequences because of their short length and variability. They were grouped into seven clusters according to the neighborhood in the CSN. Given that two peptides underrepresented clusters 1 and 5, they were fused in a cluster labeled 1-5. Thus, peptide clusters (2-4, 1-5, and singletons) were aligned independently using multiple sequence alignments (MSA), publicly available at https://www.ebi.ac.uk/Tools/msa/. Four different MSA algorithms were applied with their default parameters to determine consensus motifs within each cluster: 1) ClustalOmega v 1.2.4 [32], 2) MAFFT (Multiple Alignment using Fast Fourier Transform) v7.487 
with the iterative refinement FFT-NS-i option [33], 3) MUSCLE (Multiple Sequence Comparison by Log- Expectation) v3.8 [34], and T-Coffee (Tree-based Consistency Objective Function for Alignment Evaluation) v1.83 [35].

The resulting MSAs were employed to extract the conserved motifs by considering the consensus sequences estimation from the programs Jalview v2.11.1.4 [52], EMBOSS Cons v6.6.0 (https://www.ebi.ac.uk/Tools/msa/emboss_cons/), and Seq2Logov2.1 (http://www.cbs.dtu.dk/biotools/Seq2Logo/) [53].

\subsubsection{Alignment-Free Method}

Peptides were analyzed in STREME [36] (Sensitive, Thorough, Rapid, Enriched Motif Elicitation) to discover fixed-length patterns (ungapped motifs) that were enriched with respect to a control set generated by shuffling input peptides [52]. The analyses were performed via its webserver (https://meme-suite.org/meme/tools/streme), by considering both total peptides and by each cluster. The motif width was set between 3-5 amino acids length. STREME applies a statistical test at $p$-value threshold $=0.05$ to determine the enrichment of motifs in the input peptides compared to the control set.

\subsubsection{Motif Search in PROSITE}

Peptides were queried by the Motif Search tool (https://www.genome.jp/tools/motif/), integrated into the GenomeNet Suite (https://www.genome.jp/). PROSITE Pattern and PROSITE Profile libraries were only considered for the motif search.

\section{Results and Discussion}

\subsection{Model Selection}

\subsubsection{Network Analysis}

\subsubsection{Similarity Threshold Analysis}

Out of the set of 659 THPs retrieved from starPepDB, 627 peptides (SI1-A-I) were filtered with lower than $98 \%$ similarity by local alignment. The adequate similarity threshold was chosen before building CSN with the 627 peptides. This step is non-trivial since it is the parameter that defines the topology and networks features [54]. Hence, the appropriate cut-off for building the CSN was determined based on the variability of network parameters such as density, modularity, ACC, and singletons at different cut-off similarity values. Graphml files corresponding to the 17 CSNs are available at SI2. Table S1 shows the obtained parameters at each cut-off.

The graph of density, modularity, and ACC as a function of the similarity threshold is shown in Figure 4. The density is lower at a higher similarity threshold. ACC follows the same pattern until the 0.65 similarity threshold. By contrast, modularity increases as the similarity threshold increases, while the clustering is optimized.

A well-defined network needs a compromise among the density, modularity, and ACC parameters, but also accounts the number of outlier nodes because they are atypical peptides with particular properties. Networks with very low density display too many outliers (see Table S1), while networks with very high density show a massive connection. In both cases, information is lost and interpretation becomes difficult. According to the literature, the best density percentages are generally around $1 \%$ or $2.5 \%$ because generate high modularity but allowing an adequate understanding of the network [54]. As modularity indicates the existence of community structures, the ideal value must show an equilibrium between a non-clustered network and an artificially-clustered network due to the high modularity value. In this sense, the selected similarity threshold was 0.60 , where CSN shows the best trade-off among network parameters and connectivity: $2.3 \%$ of density, 0.47 of modularity, 0.428 of ACC, and 99 outliers ( $15.8 \%$ of overall nodes). Therefore, the giant components of the network were 528 nodes (SI1-A-II). 


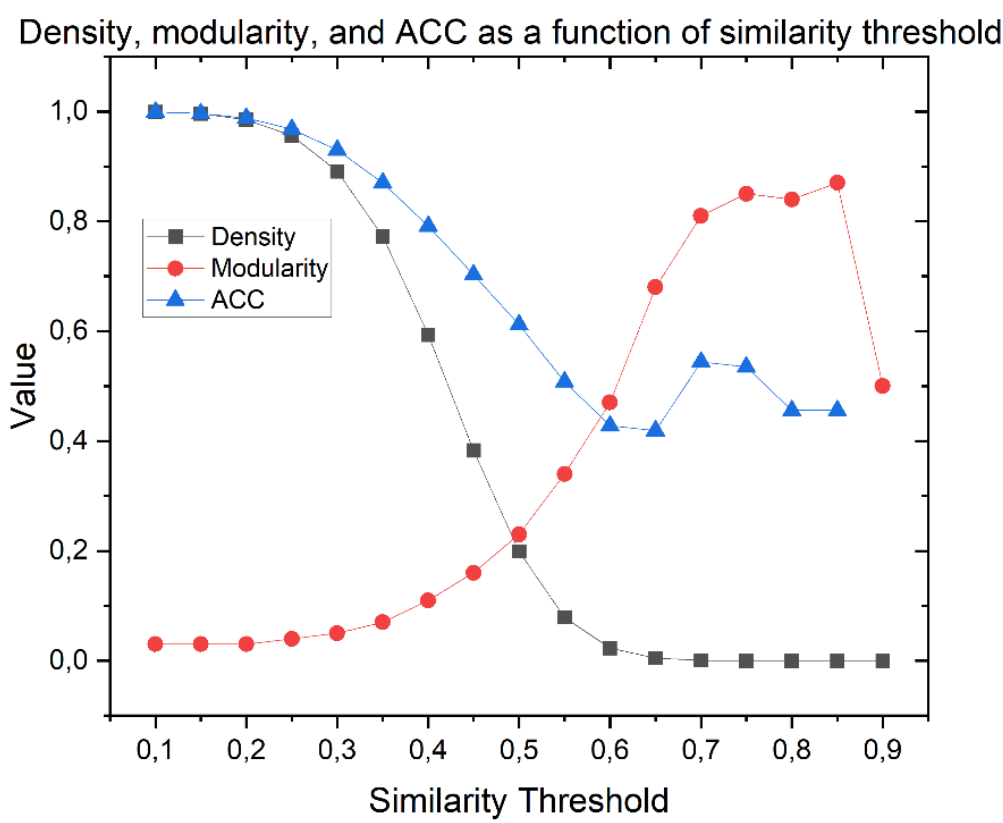

Figure 4. Density, modularity, and average clustering coefficient (ACC) as a function of similarity threshold of 627 THPs CSN.

\subsubsection{Network Characterization}

Some parameters such as density, number of clusters, modularity, average degree, ACC, and diameter were calculated and shown in Table 1 to get an overview on the giant component and outliers of the CSNs, which are represented in Figure 5 and Figure 6, respectively.

Table 1. Global networks properties of CSN of 528 nodes and outliers.

\begin{tabular}{ccccccccccc}
\hline Set* & Nodes Edges & Density & Clusters & Modularity & Average Degree & ACC & Diameter & $\begin{array}{c}\text { Nodes } \\
\text { after Sc.** } \text { after Sc. }^{* *}\end{array}$ \\
\hline THPs & 528 & 4452 & 0.023 & 10 & 0.47 & 16.864 & 0.428 & 8 & - & - \\
Outliers & 99 & 2691 & 0.891 & 3 & 0.13 & 54.364 & 0.733 & 3 & 34 & 384 \\
\hline
\end{tabular}

${ }^{*}$ Density, number of clusters, and modularity were calculated in the starPep toolbox, while average degree, ACC, and diameter in Gephi. ${ }^{*}$ Sc.: Scaffold extraction. 


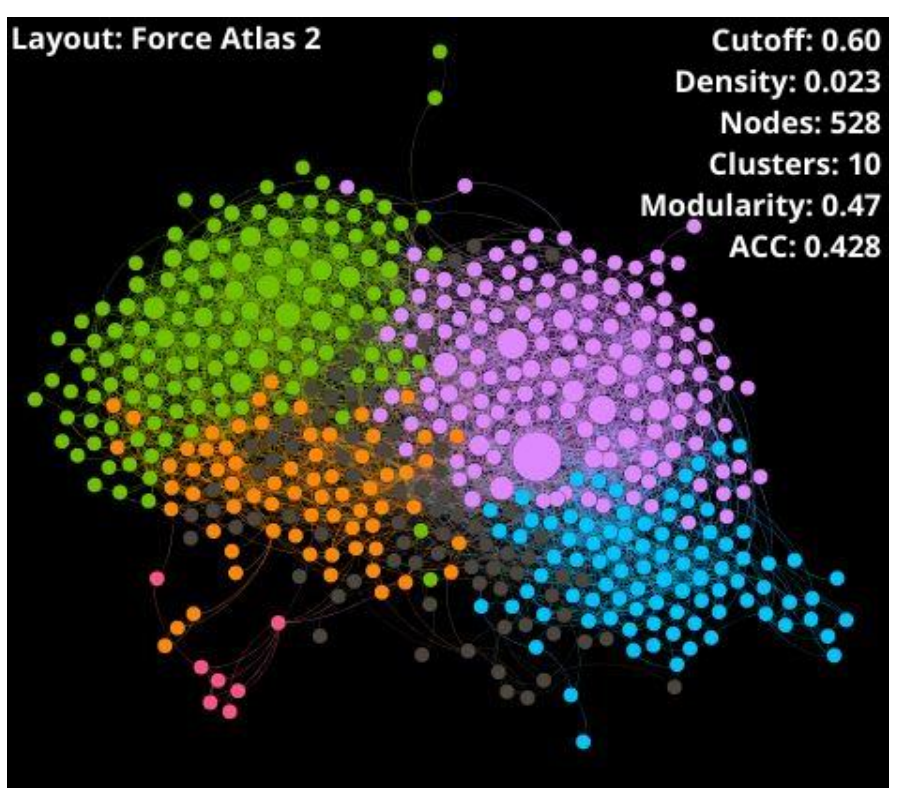

Figure 5. CSN of giant component conformed by 528 THPs retrieved from starPepDB. Nodes color represent the community (cluster), and nodes size symbolize the centrality values.

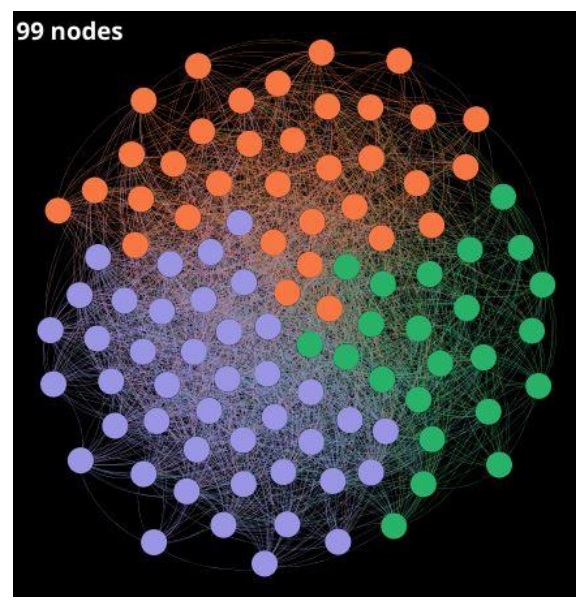

(a)

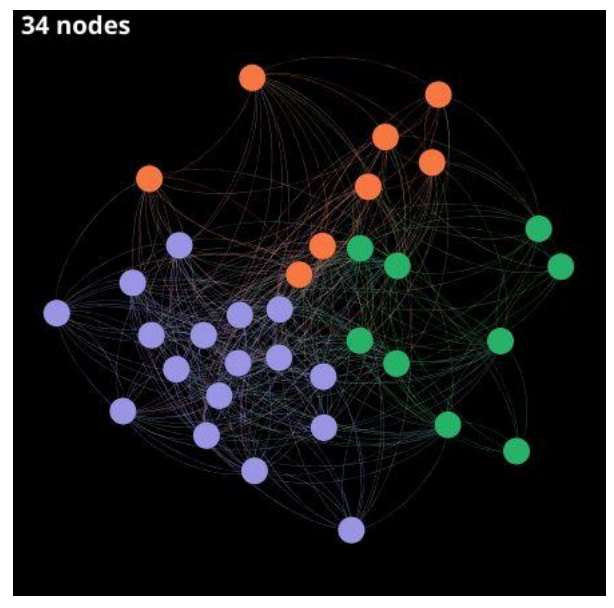

(b)

Figure 6. CSN of (a) 99 outliers with a density of 0.30 and (b) 34 remaining outliers resulted after $30 \%$ similarity extraction scaffold. Layout: Fruchterman Reingold.

Additionally, the degree of distribution of the giant components is shown in Figure 7. It gives some information about the structure of the CSN. In this case, the distribution degree is concentrated in the nodes with low vertex degrees. However, it has a tail associated with the nodes with higher vertex degrees in lower proportion. The nodes with higher degrees correspond to the most central nodes, which, as can be corroborate in Figure 5 , are in minority. 


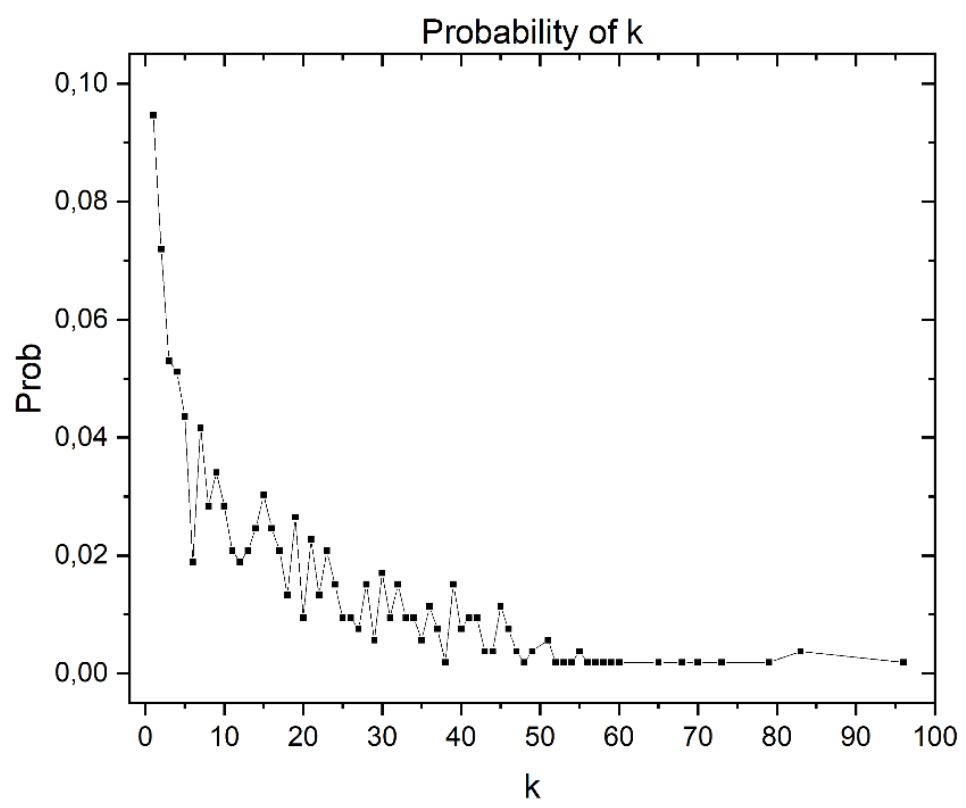

Figure 7. Degree distribution of the 528 giant components, where $\mathrm{k}$ is the vertex degree.

Outliers are relevant THPs because they present characteristics regarding 528 nodes made up the giant component; so, they are unique or atypical sequences. CSN of the 99 singletons (SI1-E) was built using 0.30 of similarity threshold (Figure 6a). Then, sequences with higher similarity than $30 \%$ by local alignment were removed based on hub-bridge centrality ranking, where 34 outliers (SI1-E-I) with unique sequences were obtained (Figure $6 b)$.

\subsubsection{Centrality Analysis and Similarity Searching.}

Centrality is the crucial parameter to build the model will be proposed to identify THPs. It allows the identification of the most influential sequences of the giant components. SI3 (Excel file) shows the normalized centrality measurements of 528 THPs. On the other hand, outliers are nodes with unique properties that enrich the influential sequences model. Therefore, both sets from centrality measurements and sets of outliers represented the chemical space of THPs and will be used as queries to perform the similarity searching against the target datasets. In total, 98 different SSMs were generated based on 22 query sets (FASTA files available at SI4) and similarity thresholds between 0.3 and 0.9 .

The predictions and performance of the 98 SSMs are shown in SI5 and SI6-A, respectively, where active and inactive labels indicates predicted THPs and non-THPs, respectively. In general, it is observed that the performance of query datasets followed the following tendency of relevance: weighted degree $>$ harmonic $>$ hub-bridge $>$ betweenness $>$ singletons (outliers). Although, the combination of query datasets from different centrality types overperforms the sets selected with only one centrality measure. The addition of the outliers set improved the performance of the combination sets since it generates the complete representation of the chemical space of THPs. Moreover, better performance was obtained using 40,50, and 60\% identity in the similarity searching.

The performance of the best nine SSMs to predict activity in Main, Small and Main90 datasets are shown in Table $2,{ }^{*} \mathrm{H}$ is the set obtained when harmonic centrality was calculated, W is the set obtained when the weighted degree was calculated, and sing is the set of 99 outliers.

Table 3 and ${ }^{*} \mathrm{H}$ is the set obtained when harmonic centrality was calculated, $\mathrm{W}$ is the set obtained when the weighted degree was calculated, and sing is the set of 99 outliers.

Table 4, respectively. It can be noticed that the best statistics were achieved using the query composed by the union of harmonic, weighted degree, in both using $60 \%$ similarity cut-off of local alignment during scaffold extraction, and the 99 outliers sets, in total 479 query sequences. Moreover, $60 \%$ was the best percentage of identity where there was a 
compromise for all statistical parameters. All statistical parameters showed values higher than 0.88 .

Table 2. Statistical analysis for the performance of the best 9 SSMs on the target Main dataset.

\begin{tabular}{ccccccccccc}
\hline $\begin{array}{c}\text { Query } \\
\text { Set }\end{array}$ & \multirow{2}{*}{ Nodes } & \% Id & Ac & $\begin{array}{c}\text { Correct } \\
\text { class }\end{array}$ & $\begin{array}{c}\text { Incorrect } \\
\text { class }\end{array}$ & $\boldsymbol{\kappa}$ & Sn & Sp & $\mathbf{P}_{\text {pos }}$ & $\mathbf{P}_{\text {neg }}$ \\
\hline H+sing & 467 & 40 & 0.933 & 1215 & 87 & 0.866 & 0.877 & 0.989 & 0.988 & 0.89 \\
& & 50 & 0.935 & 1218 & 84 & 0.871 & 0.877 & 0.994 & 0.993 & 0.89 \\
& & 60 & 0.935 & 1218 & 84 & 0.871 & 0.874 & 0.997 & 0.996 & 0.888 \\
W+sing & \multirow{2}{*}{469} & 40 & 0.934 & 1216 & 86 & 0.868 & 0.879 & 0.989 & 0.988 & 0.891 \\
& & 50 & 0.936 & 1219 & 83 & 0.873 & 0.879 & 0.994 & 0.993 & 0.891 \\
& & 60 & 0.937 & 1220 & 82 & 0.874 & 0.877 & 0.997 & 0.997 & 0.89 \\
H+W+sing & 479 & 40 & 0.942 & 1226 & 76 & 0.883 & 0.894 & 0.989 & 0.988 & 0.903 \\
& & 50 & 0.944 & 1229 & 73 & 0.888 & 0.894 & 0.994 & 0.993 & 0.904 \\
& & 60 & 0.945 & 1230 & 72 & 0.889 & 0.892 & 0.997 & 0.997 & 0.903 \\
\hline
\end{tabular}

${ }^{*} \mathrm{H}$ is the set obtained when harmonic centrality was calculated, $\mathrm{W}$ is the set obtained when the weighted degree was calculated, and sing is the set of 99 outliers.

Table 3. Statistical analysis for the performance of the best 9 SSMs on the target Small dataset.

\begin{tabular}{ccccccccccc}
\hline $\begin{array}{c}\text { Query } \\
\text { Set }\end{array}$ & \multirow{2}{*}{ Nodes } & \% Id & Ac & $\begin{array}{c}\text { Correct } \\
\text { class }\end{array}$ & $\begin{array}{c}\text { Incorrect } \\
\text { class }\end{array}$ & к & Sn & Sp & $\mathbf{P}_{\text {pos }}$ & $\mathbf{P}_{\text {neg }}$ \\
\hline H+sing & 467 & 40 & 0.917 & 860 & 78 & 0.834 & 0.838 & 0.996 & 0.995 & 0.86 \\
& & 50 & 0.916 & 859 & 79 & 0.832 & 0.836 & 0.996 & 0.995 & 0.858 \\
& & 60 & 0.914 & 857 & 81 & 0.827 & 0.832 & 0.996 & 0.995 & 0.855 \\
W+sing & \multirow{2}{*}{469} & 40 & 0.92 & 863 & 75 & 0.84 & 0.844 & 0.996 & 0.995 & 0.865 \\
& & 50 & 0.92 & 863 & 75 & 0.84 & 0.844 & 0.996 & 0.995 & 0.865 \\
& & 60 & 0.919 & 862 & 76 & 0.838 & 0.842 & 0.996 & 0.995 & 0.863 \\
H+W+sing & 479 & 40 & 0.928 & 870 & 68 & 0.855 & 0.859 & 0.996 & 0.995 & 0.876 \\
& & 50 & 0.928 & 870 & 68 & 0.855 & 0.859 & 0.996 & 0.995 & 0.876 \\
& & 60 & 0.926 & 869 & 69 & 0.853 & 0.857 & 0.996 & 0.995 & 0.875 \\
\hline
\end{tabular}

${ }^{*} \mathrm{H}$ is the set obtained when harmonic centrality was calculated, $\mathrm{W}$ is the set obtained when the weighted degree was calculated, and sing is the set of 99 outliers.

Table 4. Statistical analysis for the performance of the best 9 SSMs on the target Main90 dataset.

\begin{tabular}{|c|c|c|c|c|c|c|c|c|c|c|}
\hline $\begin{array}{c}\text { Query } \\
\text { Set }^{*}\end{array}$ & Nodes & $\%$ Id & Ac & $\begin{array}{c}\text { Correct } \\
\text { class }\end{array}$ & $\begin{array}{c}\text { Incorrect } \\
\text { class }\end{array}$ & $\kappa$ & Sn & $S p$ & $\mathbf{P}_{\text {pos }}$ & $P_{\text {neg }}$ \\
\hline \multirow[t]{3}{*}{$\mathrm{H}+$ sing } & 467 & 40 & 0.985 & 600 & 9 & 0.964 & 0.983 & 0.986 & 0.966 & 0.993 \\
\hline & & 50 & 0.99 & 603 & 6 & 0.976 & 0.983 & 0.993 & 0.983 & 0.993 \\
\hline & & 60 & 0.992 & 604 & 5 & 0.98 & 0.983 & 0.995 & 0.989 & 0.993 \\
\hline \multirow[t]{3}{*}{$W+\operatorname{sing}$} & 469 & 40 & 0.98 & 597 & 12 & 0.952 & 0.966 & 0.986 & 0.966 & 0.986 \\
\hline & & 50 & 0.984 & 599 & 10 & 0.96 & 0.966 & 0.991 & 0.977 & 0.986 \\
\hline & & 60 & 0.987 & 601 & 8 & 0.968 & 0.966 & 0.995 & 0.988 & 0.986 \\
\hline \multirow[t]{3}{*}{$\mathrm{H}+\mathrm{W}+$ sing } & 479 & 40 & 0.985 & 600 & 9 & 0.964 & 0.983 & 0.986 & 0.966 & 0.993 \\
\hline & & 50 & 0.989 & 602 & 7 & 0.972 & 0.983 & 0.991 & 0.977 & 0.993 \\
\hline & & 60 & 0.992 & 604 & 5 & 0.98 & 0.983 & 0.995 & 0.989 & 0.993 \\
\hline
\end{tabular}

${ }^{*} \mathrm{H}$ is the set obtained when harmonic centrality was calculated, $\mathrm{W}$ is the set obtained when the weighted degree was calculated, and sing is the set of 99 outliers.

The best nine SSMs were compared and ranked using the Friedman test by comparing multiple statistical metrics from each SSM on the three target datasets (details in SI6B). The best SSM was the set CSN-TH-0.60Sc-479-H+W+s-0.6-583 (479Q_0.6), named THP1, showing excellent statistical metrics $(>0.85)$ for the model (Table $2,{ }^{*} \mathrm{H}$ is the set 
obtained when harmonic centrality was calculated, $\mathrm{W}$ is the set obtained when the weighted degree was calculated, and sing is the set of 99 outliers.

Table 3, and * $\mathrm{H}$ is the set obtained when harmonic centrality was calculated, $\mathrm{W}$ is the set obtained when the weighted degree was calculated, and sing is the set of 99 outliers.

Table 4). It is composed of the union of nodes with identity lower than $60 \%$ from the global centrality harmonic with those obtained from applying weighted degree and the set of 99 outliers ( 479 nodes). The best percentage of identity used to search similarity was $60 \%$. The confusion matrices of THP1 are shown in SI6-C. It can be seen that the prediction of the model was not at random as the MCC being much greater than 0 [55].

Finally, the Friedman test of the THP1 versus the reported models used in TumorHPD [26] and THPep [28] servers revealed there is a significant difference between the models being the performance of the similarity searching methodology is superior (details in SI6-C and SI6-D). Figure 8 shows the ranking scores from the test, where THP1 is the first ranked method. Finally, Table 5 compares between the model on the three benchmarking datasets. The MCC of predictions using THP1 improved with an average of $28.76 \%$ over ML-based models.

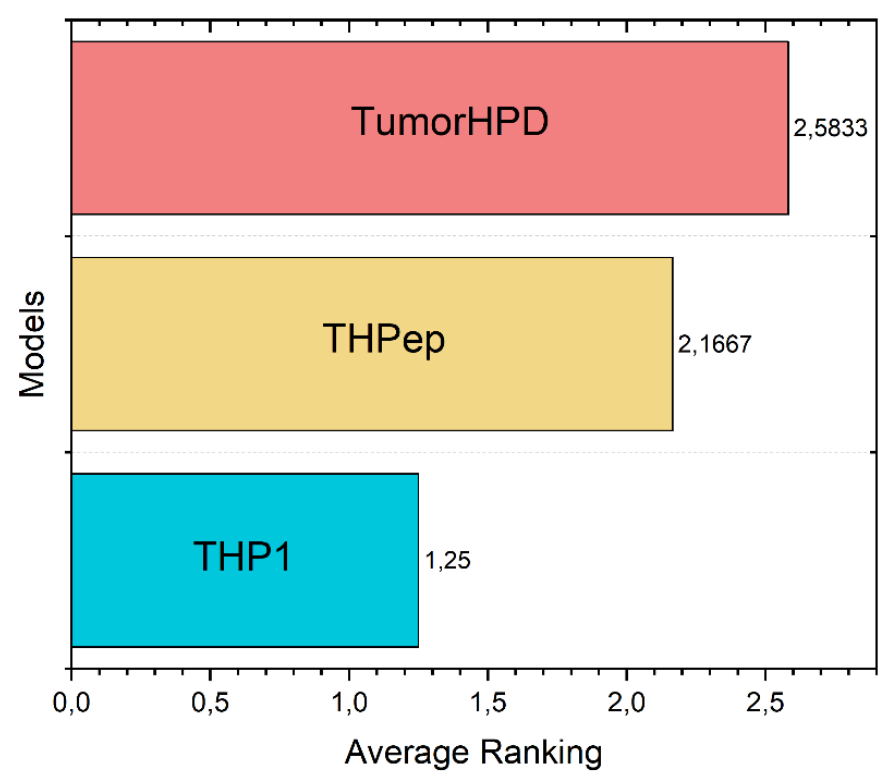

Figure 8. Ranking scores obtained in the Friedman Test. Friedman statistic (distributed according to chi-square with 2 degrees of freedom): 11.166667. P-value computed by Friedman Test: 0.00376.

Table 5. Comparison between the best SSM THP1 and the state-of-art ML model to predict tumor homing activity of benchmarking datasets.

\begin{tabular}{cccccc}
\hline Dataset & Method & Ac(\%) & Sn(\%) & Sp(\%) & MCC \\
\hline \multirow{4}{*}{ Main } & TumorHPD & 86.56 & 80.63 & 89.71 & 0.7 \\
& THPep & 86.1 & 87.07 & 85.18 & 0.72 \\
& THP1 & $\mathbf{9 4 . 4 7}$ & 89.25 & $\mathbf{9 9 . 6 6}$ & $\mathbf{0 . 8 9 4}$ \\
& TumorHPD & 81.88 & 73.13 & 90.92 & 0.65 \\
Small & THPep & 83.37 & 81.24 & 85.81 & 0.67 \\
& THP1 & 92.64 & 85.71 & 99.5 & 0.861 \\
& TumorHPD & 89.66 & 83.64 & 80.68 & 0.74 \\
Main90 & THPep & 90.8 & 91.8 & 87.97 & 0.77 \\
& THP1 & 99.18 & 98.3 & 99.54 & 0.98 \\
\hline
\end{tabular}

3.2. Identification of Potential THPS

3.2.1. Hierarchical Screening 
Starting from the 45120 AMPs contained in starPepDB, and after applying the previously explained filters and performing the similarity searching, 43 lead hits were retrieved (SI7-A). Figure 9 shows the step-by-step the hierarchical virtual screening. Until today, these repurposed sequences have not been reported any tumor homing activity.

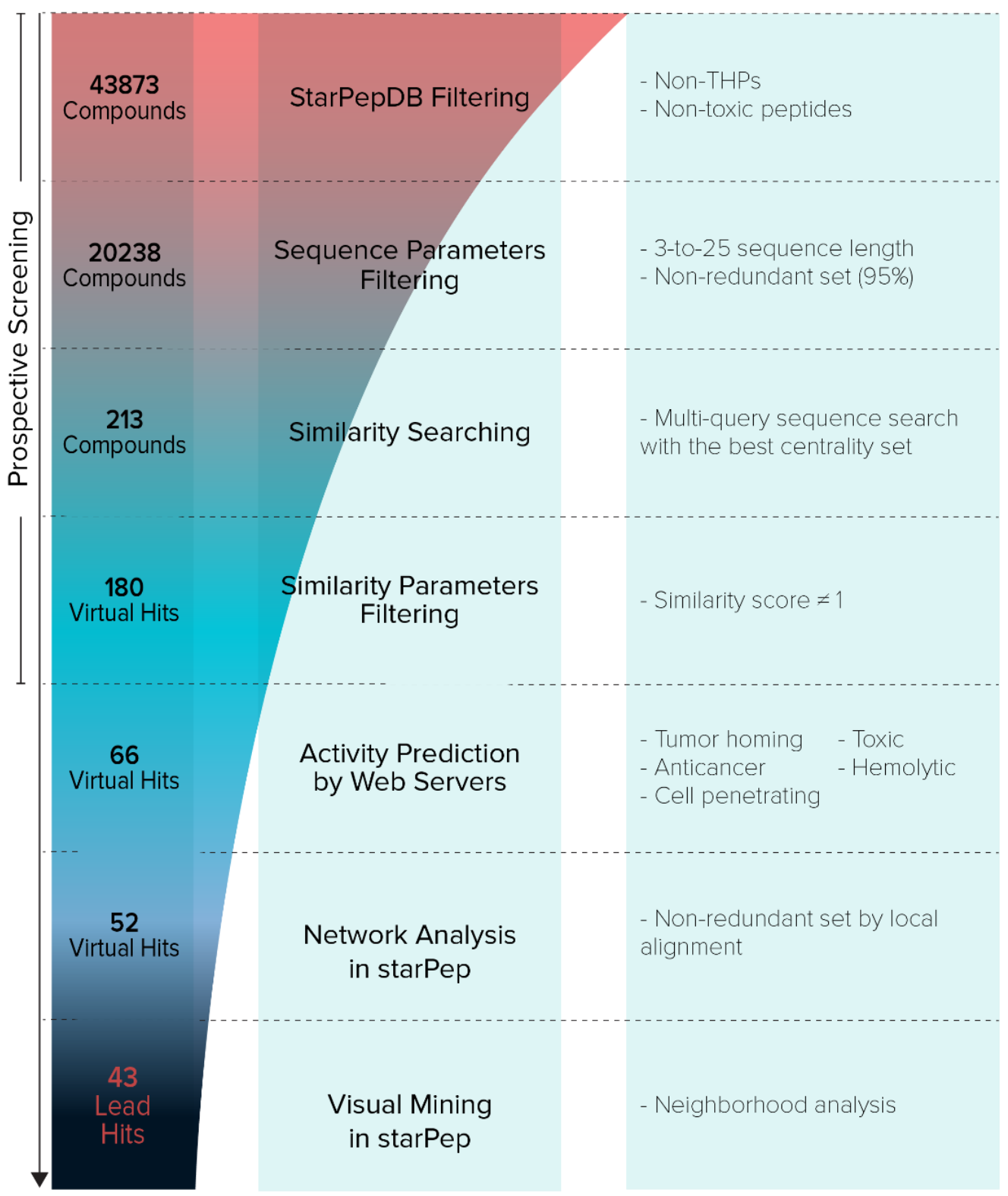

Figure 9. Hierarchical virtual screening for repurposing of peptides from starPepDB.

\subsubsection{Tumor Homing Activity Optimization}

A library of 180 sequences (SI7-B) was obtained from the optimization of 43 hits in TumorHPD. They have a higher TH score, lower toxicity, and hemolytic activity than the originals. Mutations enriched the sequences with $\mathrm{W}$ and $\mathrm{C}$ residues. Mainly, $\mathrm{G}$ and V residues from originals were mutated to $\mathrm{W}$, while $\mathrm{R}$ and $\mathrm{K}$ to $\mathrm{C}$. Studies report the presence 
of $\mathrm{W}$ contributes positively to the intracellular translocation of peptides [56]. Additionally, it was reported that $W$ enhances the stability of peptides in serum and salt [57].

Forty-one peptides from the library were prioritized by studying their CSN, where $50 \%$ scaffold extraction by local alignment was accomplished. The sequences were clustered and ranked according to the global harmonic centrality to perform the scaffold extraction. Only the most central sequences with a similarity among them lower than 50\% were kept. Forty-one sequences have higher predicted TH activity by TumorHPD than original peptides with scores between 0.39 and 1.92. Furthermore, they are anticancer and have less toxicity and hemolytic activity. 12 out of 41 sequences come from fragments of original sequences of 5,10 , and 15 lengths; 15 resulted after four punctual mutations from originals; and 14 from fragments of mutated sequences of 5, 10, and 15 lengths. Two out of 41 peptides, CNGRCGGKLA and WCAMS, are part of reported THPs, validating the novel methodology to discover potential THPs. CNGRCGGKLA is the $N$-end of the CNGRCGGKLAKLAKKLAKLAK peptide containing NGR TH motif and a disulfide bridge that gives stability. CNGRCGGKLAKLAKKLAKLAK binds to CD13 of tumor cells acting as ACP and THP [58]. At the same time, WCAMS is the C-end of KLWCAMS peptide that homes mouse B16B15b melanoma [59].

We selected the most promising 13 sequences from the 43 lead hits and were combined with the 41 optimized hits. In total, we proposed 54 peptides (SET 1, FASTA file in SI7-C) with a diverse molecular structure, low toxicity, hemolytic activity, and most of them also show potential anticancer activity (SI7-D). Among the 54 lead hits, only one sequence has the well-known NGR motif. Therefore, SET 1 is composed of new structural entities within the known structural space of the THPs.

The sequence diversity of the SET 1 was evaluated using all vs. all global alignment where pairwise sequence identities are explored. As shown in Figure 10, the 54 lead hits presents structure singularity sharing pairwise identities than $30 \%$.

\subsubsection{Motif Discovery}

As a consequence of the structural diversity of SET 1, the discovery of motifs accounting for the $\mathrm{TH}$ activity is not a straightforward task. In this sense, sensitive multiple sequence alignment (MSA) tools and alignment-free (AF) approaches (e.g., STREME) were applied to unravel new TH motifs.

The resulting 54 lead THPs were mapped onto CSN space to identify putative communities and make possible the application of MSA algorithms for motif identification. These networks communities were considered clusters containing related peptides. Finally, 6 clusters were conformed with 14, 10, 8, 4, 10, and 8 members, respectively (SI7-E). The last cluster grouped the singletons (peptides identified as atypical in the CSN).

Clustal-Omega [32], MAFFT [33], MUSCLE [34], and T-Coffee [35], which are MSA algorithms developed after the classical ClustalW, were applied, so that they can deal with hard-to-align sequences shown in each cluster, and thus to detect any conserved signature or motif. Since each MSA has implemented a different algorithm to improve alignment quality, their consideration for the estimation of consensus regions helped us identify TH motifs by using the Jalview, EMBOSS Cons and Seq2Logo programs (SI8). As the EMBOSS Cons, gives a more legible output, only displaying high scored amino acids/positions (capital letters), less scored but positive residues (lower-case letters), and non-consensus positions $(\mathrm{x})$, were selected as the primary source to set consensus/conserved regions. The non-consensus positions were estimated using default parameters by visual inspection of the corresponding positions in the Jalview program [52] and the Seq2Logo [53]. Table 6 depicts the consensus motifs, unraveled by each MSA algorithm. 


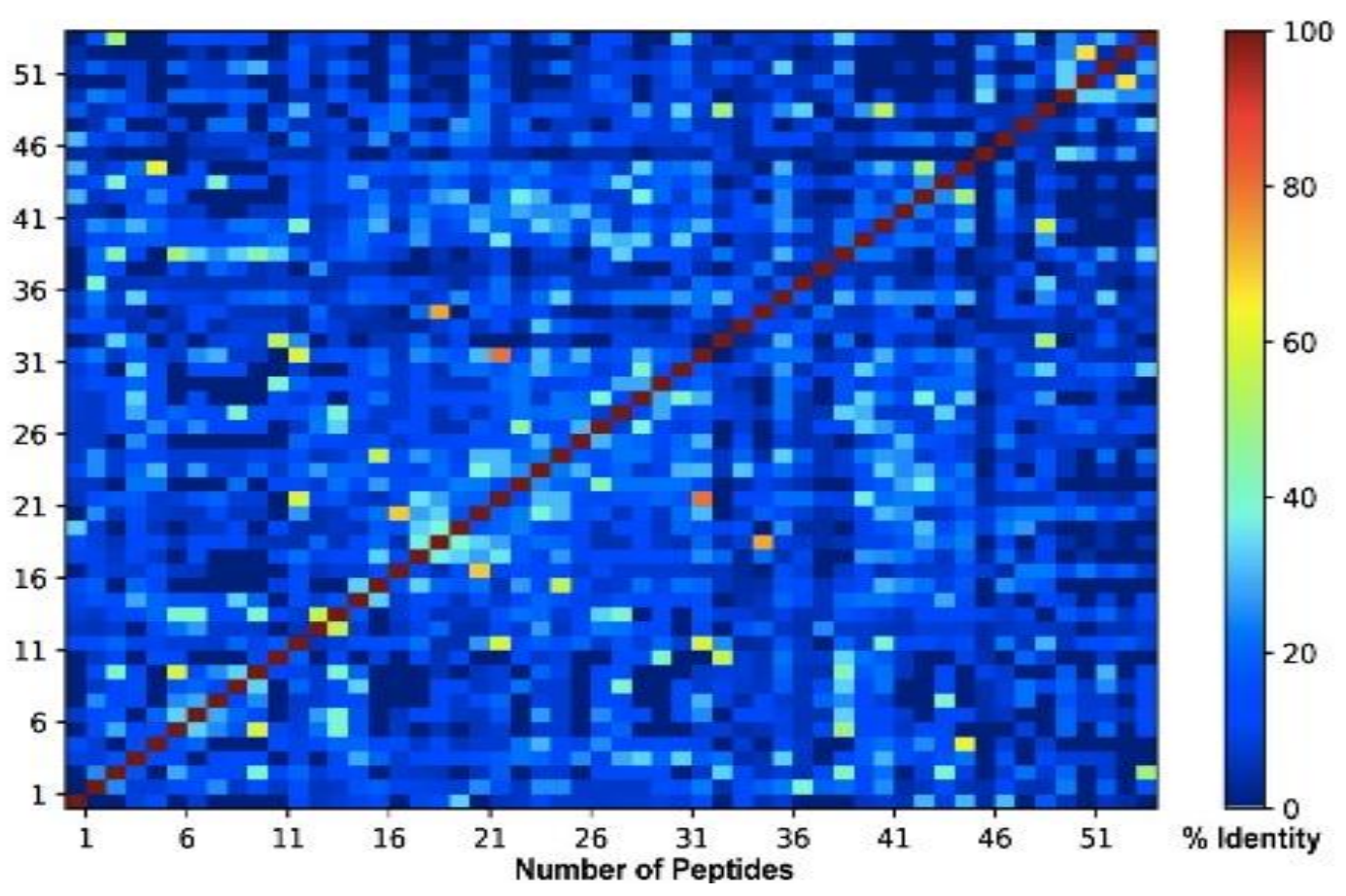

(a)

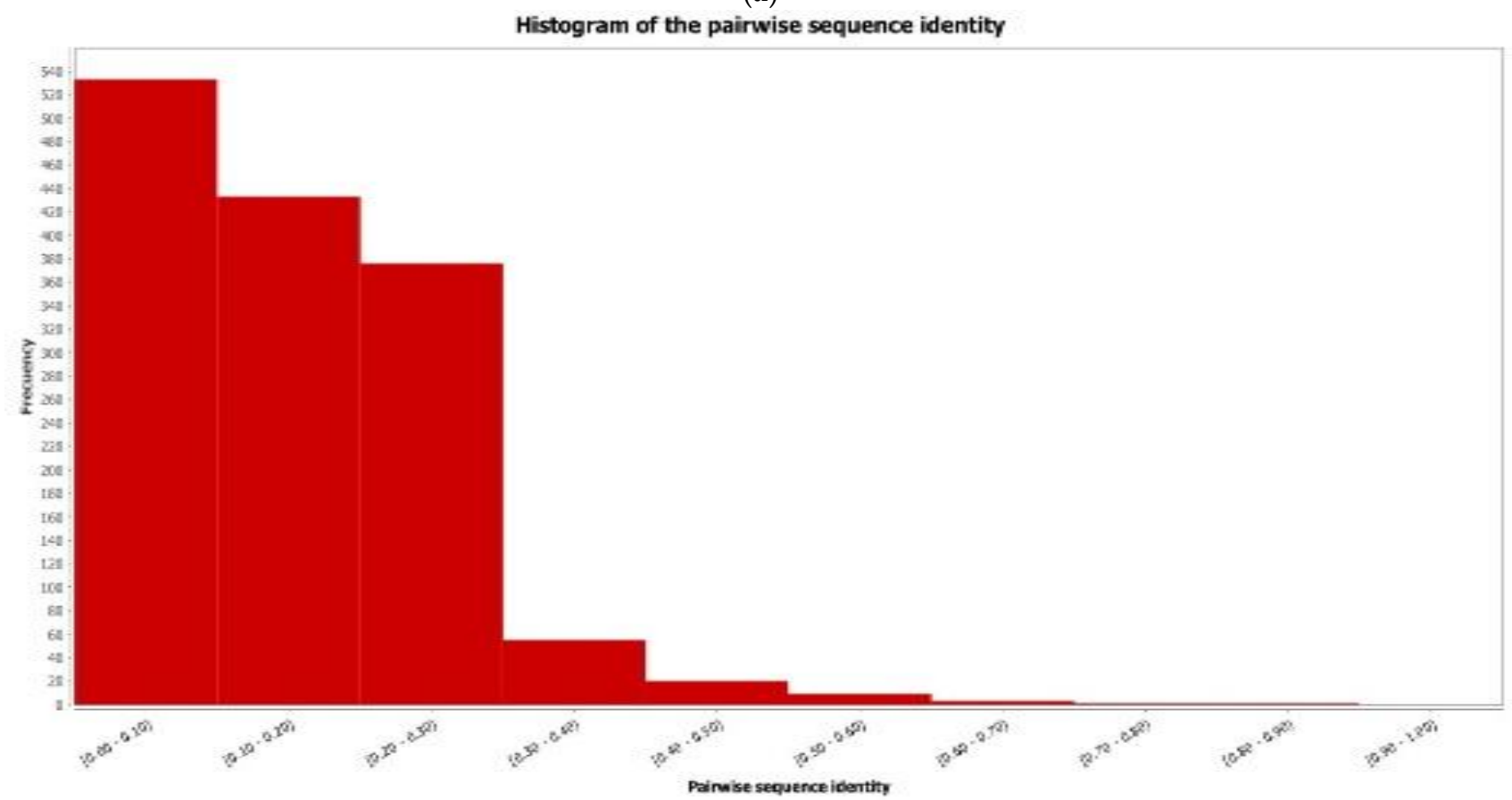

(b)

Figure 10. (a) Heat map and (b) histogram of pairwise sequence identity of SET 1 (54 lead compounds).

None of the motifs found by MSA have been reported as TH motifs (Table 6). However, one of the motifs from No.3 CxxxR, CGGCR, contains the CXXC motif, which is the active site of thioredoxin (Trx), a relevant protein in mammalian cells that act as an antioxidant and participates in programmed cell death inhibition and cell growth, commonly used as a target for cancer treatments [60,61]. Moreover, CWKG (No.5) is contained in a nanoscale molecular platform used as drug delivery system in chemotherapy to enhance the conjugation of mitomycin $C$ to the carrier [62].

Table 6. Discovered motifs by Multiple Sequence Alignment (MSA). 


\begin{tabular}{|c|c|c|c|c|c|c|}
\hline No & Motif & EMBOSS Consensus & Cluster & Cluster size & Frequency* & MSA Method \\
\hline \multirow{2}{*}{1} & \multirow{2}{*}{ wwW } & wwW & \multirow{2}{*}{2} & \multirow{2}{*}{14} & \multirow{2}{*}{$1 /(1)$} & CLUSTALW-O \\
\hline & & $\mathrm{xxW}$ & & & & MAFFT \\
\hline 2 & $\mathrm{C}[\mathrm{fl}][\mathrm{rg}][\mathrm{vl}] \mathrm{rW}$ & CxxxrW & \multirow{2}{*}{3} & \multirow{2}{*}{10} & \multirow{2}{*}{$0 /(0)$} & MAFFT \\
\hline 3 & C[gpi][gs]cR & CxxxR & & & & MUSCLE \\
\hline \multirow{3}{*}{4} & \multirow{3}{*}{ [rkl]GLC } & RGlc & \multirow{3}{*}{4} & \multirow{3}{*}{8} & \multirow{3}{*}{$0 /(0)$} & CLUSTALW-O \\
\hline & & kGLC & & & & MAFFT \\
\hline & & $x G L c$ & & & & MUSCLE \\
\hline \multirow{3}{*}{5} & \multirow{3}{*}{$c[w p] k G$} & & \multirow{3}{*}{$1+5$} & \multirow{3}{*}{4} & $0 /(0)$ & CLUSTALW-O \\
\hline & & cWkG & & & $0 /(0)$ & MUSCLE \\
\hline & & cxkG & & & $0 /(1)$ & T-Coffee \\
\hline 6 & Not Found & Non-consensus & 6 & 10 & $0 /(0)$ & $\begin{array}{c}\text { CLUSTALW-O } \\
\text { MUSCLE } \\
\text { MAFFT } \\
\text { T-Coffee }\end{array}$ \\
\hline 7 & $1[\mathrm{rp}][\mathrm{cw}] \mathrm{c}$ & $1 \times x c$ & Singletons & 8 & $0 /(0)$ & MUSCLE \\
\hline
\end{tabular}

On the other hand, the AF approach STREME was used to find unaligned patterns ranging 3-5 aa length within the overall 54 peptides and each peptide cluster. STREME has been recently reported as the most accurate and sensitive algorithm among its competing state-of-art partners [36]. Unlike previous algorithms [63-65], STREME uses a position weight matrix (PWM) to count position matches efficiently for a motif candidate against a Markov model built up from shuffling the same input set (control sequences). Table 7 displays the enriched motifs discriminating the 54 lead peptides against the control sequences. The same search was also performed by considering each cluster content. Motifs appearing in more than $20 \%$ of the query sequences are listed according to their statistical significance (score).

One of the motifs discovered by STREME had been reported as tumor homing, WRP interacting with VEGF-C [66,67]. Other found motifs have been reported but not as TH, such as WRPW, PRW, WKG, and PSHL. WRPW is the binding site of the 7 Enhancer of split E(spl) basic helix-loop-helix (bHLH) protein and the Hairy protein to the corepressor protein Groucho-TLE via WD40 domain [68]. PRW is part of a biocatalyst, which is conjugated to a lipid by an ester or amide bond [69]. WKG is a ribosomally synthesized and post-translationally modified peptide [70] and PSHL is a tetrapeptide that affects HIV-1 protease (PR) [71].

Lastly, 54 lead THPs were queried against PROSITE's pattern and profile databases using the search engine Motif Search of the GenomeNet suite [72]. Only two query peptides, which are shown in Table 8, had significant matches with motifs found in Gonadotropin-releasing hormones $(\mathrm{GnRH})$ and Bombesin-like peptides.

These two peptide signatures and their receptors are involved in neuroendocrine signaling pathways associated with physiological states and tumors. GnRH is the hypothalamic decapeptide that plays a crucial role controlling women's reproductive cycle. GnRH binds to specific receptors on the pituitary gonadotrophic cells, but it also is expressed in other reproductive organs, e.g. ovaries, and tumors derived from the ovaries. It has been shown GnRH is involved in the ovarian cancer regulation proliferation and metastasis either by indirect signaling pathway or direct interaction with the GnRH receptors placed at the surface of ovarian cancer cells [73].

Table 7. Discovered Motifs by STREME. 


\begin{tabular}{|c|c|c|c|c|c|c|c|c|}
\hline No & Motif & Cluster & $\begin{array}{l}\text { Cluster } \\
\text { size }\end{array}$ & $\begin{array}{c}\text { Matches in } \\
\text { positive } \\
\text { seqs. }\end{array}$ & $\begin{array}{c}\text { Matches } \\
\text { in control } \\
\text { seqs. }\end{array}$ & Sites $(\%)$ & Score & $\begin{array}{c}\text { Fre- } \\
\text { quency* }\end{array}$ \\
\hline 1 & WRP & \multirow{4}{*}{2} & \multirow{4}{*}{14} & 7 & 1 & 50 & $1.6 \mathrm{e}-002$ & $5 /(5)$ \\
\hline 2 & WVL & & & 5 & 1 & 35.7 & $8.2 \mathrm{e}-002$ & $0 /(0)$ \\
\hline 3 & WS[YR] & & & 3 & 0 & 21.4 & $1.1 \mathrm{e}-001$ & $1 /(1) \mathrm{Y}$ \\
\hline 4 & WWWM & & & 3 & 0 & 21.4 & $1.1 \mathrm{e}-001$ & $0 /(0)$ \\
\hline 5 & CFRV & \multirow{3}{*}{3} & \multirow{3}{*}{10} & 3 & 0 & 30 & $1.1 \mathrm{e}-001$ & $1 /(1)$ \\
\hline 6 & HWK & & & 2 & 0 & 20 & $2.4 \mathrm{e}-001$ & $0 /(0)$ \\
\hline 7 & PRW & & & 2 & 0 & 20 & $2.4 \mathrm{e}-001$ & $3 /(3)$ \\
\hline 8 & CN[WG] & \multirow{3}{*}{4} & \multirow{3}{*}{8} & 3 & 0 & 37.5 & $1.0 \mathrm{e}-001$ & $34 /(32) \mathrm{G}$ \\
\hline 9 & WARG & & & 3 & 0 & 37.5 & $1.0 \mathrm{e}-001$ & $0 /(0)$ \\
\hline 10 & GIC & & & 2 & 0 & 25.0 & $2.3 e-001$ & $5 /(4)$ \\
\hline 11 & WKG & $1-5$ & 4 & 3 & 1 & 75.0 & $2.4 \mathrm{e}-001$ & $0 /(0)$ \\
\hline 12 & KNKHK & \multirow{2}{*}{6} & \multirow{2}{*}{10} & 3 & 0 & 30.0 & $1.1 \mathrm{e}-001$ & $0 /(0)$ \\
\hline 13 & PSHL & & & 3 & 0 & 30.0 & $1.1 \mathrm{e}-001$ & $0 /(0)$ \\
\hline 14 & LRLRI & \multirow{2}{*}{ Singletons } & \multirow{2}{*}{8} & 2 & 0 & 25.0 & $2.3 e-001$ & $1 /(1)$ \\
\hline 15 & $\mathrm{CC}[\mathrm{CQ}]$ & & & 3 & 1 & 37.5 & $2.8 \mathrm{e}-001$ & $0 /(0)$ \\
\hline 16 & LSP & \multirow{3}{*}{$\begin{array}{l}\text { All se- } \\
\text { quences }\end{array}$} & \multirow{3}{*}{54} & 11 & 1 & 20.4 & $3.4 \mathrm{e}-003$ & $3 /(3)$ \\
\hline 17 & WSYG & & & 7 & 0 & 13.0 & $8.2 \mathrm{e}-003$ & $0 /(0)$ \\
\hline 18 & WRPW & & & 5 & 0 & 9.3 & $3.2 \mathrm{e}-002$ & $2 /(2)$ \\
\hline
\end{tabular}

Table 8. Motifs found in PROSITE.

\begin{tabular}{cccccccc}
\hline No & Motif Found & Hit Peptide & $\begin{array}{c}\text { Acces- } \\
\text { sion }\end{array}$ & Match with & Signature & $\begin{array}{c}\text { Related } \\
\text { Seqs. }\end{array}$ & $\begin{array}{c}\text { Frequency } \\
\text { Sonadotropin- }\end{array}$ \\
\hline $\mathbf{1}$ & QHWSYGLRPG & $\begin{array}{c}\text { star- } \\
\text { Pep_07237 }\end{array}$ & PS00473 & Q[HY][FYW]Sx(4)PG & $\begin{array}{c}\text { releasing hor- } \\
\text { mones }\end{array}$ & 67 & $1 /(1) Q H W S Y$ \\
\hline 2 & WARGHFM & $\begin{array}{c}\text { star- } \\
\text { Pep_10020 }\end{array}$ & PS00257 & WAxG[SH][LF]M & $\begin{array}{c}\text { Bombesin-like } \\
\text { peptides }\end{array}$ & 36 & $0 /(0)$ \\
\hline
\end{tabular}

*Taken from TumorHoPe (outside parenthesis), and starPepDB (inside parenthesis).

Bombesin-like peptides were initially discovered from the frog skin, where they are secreted from cutaneous glands as a means of communication and defense. They were later found to be widely distributed in mammalian neural and endocrine cells represented by the neuromedin B (NMB) and the gastrin-releasing peptide (GRP), respectively. Bombesin-like peptide receptors are $G$ protein-coupled and have seven membrane-spanning domains, so they are involved in signal transduction pathways [74]. Growing evidence shows that bombesin-like peptides and receptors play essential roles in physiological conditions and diseases. An abnormal expression of bombesin receptors has been observed in several types of tumors, which has motivated the development of more specific and safer bombesin-derivatives for tumors diagnosis and therapy [75].

The motif search by using different approaches may render a diversity of outcomes. However, some hits shared by different search approaches can support the reliability of the findings. For example, one motif WSY found by the PROSITE search was also encountered by STREME, an algorithm that works regardless of database and sequence similarity. Some of the motifs estimated by MSA algorithms were also identified by the AF approach STREME such as WWW and WKG. All motifs were searched against TH databases, TumorHoPe, and starPepDB to discriminate the possible new signatures from the existing ones. New motifs appear at very low frequency within THPs (last column of Table 6, Table 7 and Table 8), except CNG found by STREME, which appears 34 times in TumorHoPe and 32 in starPepDB. However, CNG has not been reported as a TH motif. 


\title{
4. Conclusions
}

In this study, a novel methodology based on network science and similarity searching was introduced to explore the chemical space of THPs and discover potential THPs from known AMPs. Statistically, the performance of the strategy's performance transcends current supervised ML approaches used in THPs predictions, demonstrating the potential of this approach. Hence, in silico predictions using the model based on representative THPs in conjunction with TumorHPD and THPep servers give high reliability to discover potential THPs. As a result, 54 lead compounds are repurposed as potential from AMPs. In the set, novel motifs with promising tumor homing activity are proposed.

The good performance of the methodology for predicting peptide activity based on similarity searching and network science suggests its application for the prediction of other endpoints in peptides, e.g. antibacterial activity, toxicity, hemolytic, or anticancer. Our models and pipeline are freely available through the starPep toolbox software at http://mobiosd-hub.com/starpep.

Supplementary Materials: The Supporting Information is available free of charge at Zenodo. https://doi.org/10.5281/zenodo.5935872

\begin{abstract}
Author Contributions: R, M. worked on the datasets extraction and curation, designed the experiments, performed SAR analysis, and performed the virtual screening as well as drafted the initial manuscript. M-P, Y. worked on conceptualizing of the complex network and similarity searching methods, supervised the applications and prepared the manuscript. R, H., A-C, G., and A, A. worked mainly on the motif discovery analysis and drafted the initial manuscript. A-M, L. and M$\mathrm{R}, \mathrm{F}$. worked primarily in the implementation of the complex network and similarity searching module and performed SAR and statistical analysis. All authors read, reviewed, and approved the final manuscript.
\end{abstract}

Funding: Declared none.

Institutional Review Board Statement: Not applicable.

Informed Consent Statement: Not applicable.

Data Availability Statement: The starPep toolbox software and the respective user manual, as well as SSMs, are freely available online at http://mobiosd-hub.com/starpep.

Acknowledgments: Yovani Marrero-Ponce (M-P, Y) thanks to the program Profesor coinvitado for a post-doctoral fellowship to work at Valencia University in 2020. M-P, Y. acknowledge the support from Collaboration Grant 2019-2020 (Project ID16897) and Med Grant 2019-2020 (Project ID16905). A-C, G. and A, A. were supported by national funds through FCT - Foundation for Science and Technology within the scope of UIDB/04423/2020 and UIDP/04423/2020. Hortensia Rodríguez (R, H.) and Maylin Romero (R, M.) acknowledge the support of Yachay Tech internal Project "Therapeutic Peptides with biological activity" (REG-INV-18-03244).

Conflicts of Interest: The authors declare no conflict of interest.

\section{References}

1. World Health Organization Cancer Available online: https://www.who.int/health-topics/cancer\#tab=tab_1.

2. Hoskin, D.W.; Ramamoorthy, A. Studies on anticancer activities of antimicrobial peptides. Biochim. Biophys. Acta - Biomembr. 2008, 1778, 357-375, doi:10.1016/j.bbamem.2007.11.008.

3. Miller, K.D.; Nogueira, L.; Mariotto, A.B.; Rowland, J.H.; Yabroff, K.R.; Alfano, C.M.; Jemal, A.; Kramer, J.L.; Siegel, R.L. Cancer treatment and survivorship statistics, 2019. CA. Cancer J. Clin. 2019, 69, 363-385, doi:10.3322/caac.21565.

4. Gatti, L.; Zunino, F. Overview of Tumor Cell Chemoresistance Mechanisms. In Chemosensitivity; Humana Press: New Jersey, 2005; Vol. 111, pp. 127-148.

5. de la Torre, B.G.; Albericio, F. Peptide therapeutics 2.0. Molecules 2020, 25, 2019-2021, doi:10.3390/molecules25102293.

6. Lau, J.L.; Dunn, M.K. Therapeutic peptides: Historical perspectives, current development trends, and future directions. Bioorg. 
Med. Chem. 2018, 26, 2700-2707, doi:10.1016/j.bmc.2017.06.052.

7. Albericio, F.; Kruger, H.G. Therapeutic peptides. Future Med. Chem. 2012, 4, 1527-1531, doi:10.4155/fmc.12.94.

8. Ladner, R.C.; Sato, A.K.; Gorzelany, J.; De Souza, M. Phage display-derived peptides as therapeutic alternatives to antibodies. Drug Discov. Today 2004, 9, 525-529, doi:10.1016/S1359-6446(04)03104-6.

9. Vlieghe, P.; Lisowski, V.; Martinez, J.; Khrestchatisky, M. Synthetic therapeutic peptides: science and market. Drug Discov. Today 2010, 15, 40-56, doi:10.1016/j.drudis.2009.10.009.

10. Loffet, A. Peptides as drugs: Is there a market? J. Pept. Sci. 2002, 8, 1-7, doi:10.1002/psc.366.

11. Segura-Campos, M.; Chel-Guerrero, L.; Betancur-Ancona, D.; Hernandez-Escalante, V.M. Bioavailability of bioactive peptides. Food Rev. Int. 2011, 27, 213-226, doi:10.1080/87559129.2011.563395.

12. Wu, D.; Gao, Y.; Qi, Y.; Chen, L.; Ma, Y.; Li, Y. Peptide-based cancer therapy: Opportunity and challenge. Cancer Lett. 2014, 351, 13-22, doi:10.1016/j.canlet.2014.05.002.

13. Wei, G.; Wang, Y.; Huang, X.; Hou, H.; Zhou, S. Peptide-Based Nanocarriers for Cancer Therapy. Small Methods 2018, 2, 116, doi:10.1002/smtd.201700358.

14. Tesauro, D.; Accardo, A.; Diaferia, C.; Milano, V.; Guillon, J.; Ronga, L.; Rossi, F. Peptide-Based Drug-Delivery Systems in Biotechnological Applications: Recent Advances and Perspectives. Molecules 2019, 24, 351, doi:10.3390/molecules24020351.

15. Ruoslahti, E. Tumor penetrating peptides for improved drug delivery. Adv. Drug Deliv. Rev. 2017, 110-111, 3-12, doi:10.1016/j.addr.2016.03.008.

16. Khandia, R.; Sachan, S.; K. Munjal, A.; Tiwari, R.; Dhama, K. Tumor Homing Peptides: Promising Futuristic Hope for Cancer Therapy. In Topics in Anti-Cancer Research; BENTHAM SCIENCE PUBLISHERS, 2016; pp. 43-86.

17. Laakkonen, P.; Vuorinen, K. Homing peptides as targeted delivery vehicles. Integr. Biol. 2010, 2, 326-337, doi:10.1039/c0ib00013b.

18. Elsabahy, M.; Shrestha, R.; Clark, C.; Taylor, S.; Leonard, J.; Wooley, K.L. Multifunctional hierarchically assembled nanostructures as complex stage-wise dual-delivery systems for coincidental yet differential trafficking of siRNA and paclitaxel. Nano Lett. 2013, 13, 2172-2181, doi:10.1021/nl4006645.

19. Kolonin, M.G.; Bover, L.; Sun, J.; Zurita, A.J.; Do, K.A.; Lahdenranta, J.; Cardó-Vila, M.; Giordano, R.J.; Jaalouk, D.E.; Ozawa, M.G.; et al. Ligand-directed surface profiling of human cancer cells with combinatorial peptide libraries. Cancer Res. 2006, 66, 34-40, doi:10.1158/0008-5472.CAN-05-2748.

20. Peletskaya, E.N.; Glinsky, V. V.; Glinsky, G. V.; Deutscher, S.L.; Quinn, T.P. Characterization of peptides that bind the tumorassociated Thomsen-Friedenreich antigen selected from bacteriophage display libraries. J. Mol. Biol. 1997, 270, 374-384, doi:10.1006/jmbi.1997.1107.

21. Wang, F.; Li, Y.; Shen, Y.; Wang, A.; Wang, S.; Xie, T. The functions and applications of RGD in tumor therapy and tissue engineering. Int. J. Mol. Sci. 2013, 14, 13447-13462, doi:10.3390/ijms140713447.

He, X.; Na, M.; Kim, J.-S.; Lee, G.-Y.; Park, J.Y.; Hoffman, A.S.; Nam, J.; Han, S.; Sim, G.Y.; Oh, Y.; et al. A Novel Peptide Probe for Imaging and Targeted Delivery of Liposomal Doxorubicin to Lung Tumor. Mol. Pharm. 2011, 8, 430-438, doi:10.1021/mp100266g.

23. Nazemian, M.; Hojati, V.; Zavareh, S.; Madanchi, H.; Hashemi-Moghaddam, H. Immobilized Peptide on the Surface of Poly 1-DOPA/Silica for Targeted Delivery of 5-Fluorouracil to Breast Tumor. Int. J. Pept. Res. Ther. 2020, 26, 259-269, doi:10.1007/s10989-019-09834-2.

24. Wu, C.-H.; Liu, I.-J.; Lu, R.-M.; Wu, H.-C. Advancement and applications of peptide phage display technology in biomedical science. J. Biomed. Sci. 2016, 23, 8, doi:10.1186/s12929-016-0223-x.

25. Cui, W.; Aouidate, A.; Wang, S.; Yu, Q.; Li, Y.; Yuan, S. Discovering Anti-Cancer Drugs via Computational Methods. Front. Pharmacol. 2020, 11, 1-14, doi:10.3389/fphar.2020.00733.

26. Sharma, A.; Kapoor, P.; Gautam, A.; Chaudhary, K.; Kumar, R.; Chauhan, J.S.; Tyagi, A.; Raghava, G.P.S. Computational approach for designing tumor homing peptides. Sci. Rep. 2013, 3, 1-7, doi:10.1038/srep01607. 
27. Kapoor, P.; Singh, H.; Gautam, A.; Chaudhary, K.; Kumar, R.; Raghava, G.P.S. TumorHoPe: A Database of Tumor Homing Peptides. PLoS One 2012, 7, doi:10.1371/journal.pone.0035187.

28. Shoombuatong, W.; Schaduangrat, N.; Pratiwi, R.; Nantasenamat, C. THPep: A machine learning-based approach for predicting tumor homing peptides. Comput. Biol. Chem. 2019, 80, 441-451, doi:10.1016/j.compbiolchem.2019.05.008.

29. Aguilera-Mendoza, L.; Marrero-Ponce, Y.; Beltran, J.A.; Tellez Ibarra, R.; Guillen-Ramirez, H.A.; Brizuela, C.A. Graph-based data integration from bioactive peptide databases of pharmaceutical interest: toward an organized collection enabling visual network analysis. Bioinformatics 2019, 35, 4739-4747, doi:10.1093/bioinformatics/btz260.

30. Charoenkwan, P.; Chiangjong, W.; Nantasenamat, C.; Moni, M.A.; Lio', P.; Manavalan, B.; Shoombuatong, W. SCMTHP: A New Approach for Identifying and Characterizing of Tumor-Homing Peptides Using Estimated Propensity Scores of Amino Acids. Pharmaceutics 2022, 14, 122, doi:10.3390/pharmaceutics14010122.

31. Aguilera-Mendoza, L.; Marrero-Ponce, Y.; García-Jacas, C.R.; Chavez, E.; Beltran, J.A.; Guillen-Ramirez, H.A.; Brizuela, C.A. Automatic construction of molecular similarity networks for visual graph mining in chemical space of bioactive peptides: an unsupervised learning approach. Sci. Rep. 2020, 10, 18074, doi:10.1038/s41598-020-75029-1.

32. Sievers, F.; Wilm, A.; Dineen, D.; Gibson, T.J.; Karplus, K.; Li, W.; Lopez, R.; McWilliam, H.; Remmert, M.; Söding, J.; et al. Fast, scalable generation of high-quality protein multiple sequence alignments using Clustal Omega. Mol. Syst. Biol. 2011, 7, doi:10.1038/msb.2011.75.

33. Katoh, K.; Misawa, K.; Kuma, K.I.; Miyata, T. MAFFT: A novel method for rapid multiple sequence alignment based on fast Fourier transform. Nucleic Acids Res. 2002, 30, 3059-3066, doi:10.1093/nar/gkf436.

34. Edgar, R.C. MUSCLE: Multiple sequence alignment with high accuracy and high throughput. Nucleic Acids Res. 2004, 32, 1792-1797, doi:10.1093/nar/gkh340.

35. Notredame, C.; Higgins, D.G.; Heringa, J. T-coffee: A novel method for fast and accurate multiple sequence alignment. J. Mol. Biol. 2000, 302, 205-217, doi:10.1006/jmbi.2000.4042.

36. Bailey, T.L. STREME: accurate and versatile sequence motif discovery. Bioinformatics 2021, doi:10.1093/bioinformatics/btab203.

37. Contreras-Torres, E.; Marrero-Ponce, Y.; E. Terán, J.; R. García-Jacas, C.; A. Brizuela, C.; Carlos Sánchez-Rodríguez, J. MuLiMs-MCoMPAs: A Novel Multiplatform Framework to Compute Tensor Algebra-Based Three-Dimensional Protein Descriptors. J. Chem. Inf. Model. 2019, 0, doi:10.1021/acs.jcim.9b00629.

38. Fruchterman, T.M.J.; Reingold, E.M. Graph Drawing by. Force-Directed Placement, Software-Practice Exp. 1991, vol, 21no11pp1129-1164.

39. Blondel, V.D.; Guillaume, J.-L.; Lambiotte, R.; Lefebvre, E. Fast unfolding of communities in large networks. J. Stat. Mech. Theory Exp. 2008, 2008, P10008, doi:10.1088/1742-5468/2008/10/P10008.

40. Reigosa, M.J.; Gonzalez, L.; Sanches-Moreiras, A.; Duran, B.; Puime, D.; Fernadez, D.A.; Bolano, J.C. Comparison of physiological effects of allelochemicals and commercial herbicides. Allelopath. J. 2001, 8, 211-220.

41. Bastian, M.; Heymann, S.; Jacomy, M. Gephi: An open source software for exploring and manipulating networks. BT International AAAI Conference on Weblogs and Social. Int. AAAI Conf. Weblogs Soc. Media 2009, 361-362.

42. Shoombuatong, W.; Schaduangrat, N.; Nantasenamat, C. UNRAVELING THE BIOACTIVITY OF ANTICANCER PEPTIDES AS. EXCLI J. 2018, 17, 734-752, doi:10.17179/excli2018-1447 This.

43. Willett, P. Similarity-based virtual screening using 2D fingerprints. Drug Discov. Today 2006, 11, 1046-1053, doi:10.1016/j.drudis.2006.10.005.

44. Triguero, I.; González, S.; Moyano, J.M.; García, S.; Alcalá-Fdez, J.; Luengo, J.; Fernández, A.; del Jesús, M.J.; Sánchez, L.; Herrera, F. KEEL 3.0: An Open Source Software for Multi-Stage Analysis in Data Mining. Int. J. Comput. Intell. Syst. 2017, 10, 1238, doi:10.2991/ijcis.10.1.82.

45. Iman, R.L.; Davenport, J.M. Approximations of the critical region of the Friedman statistic. Commun. Stat. - Theory Methods 1980, 9, 571-595, doi:10.1080/03610928008827904. 
46. Lee, W.H.; Loo, C.Y.; Ghadiri, M.; Leong, C.R.; Young, P.M.; Traini, D. The potential to treat lung cancer via inhalation of repurposed drugs. Adv. Drug Deliv. Rev. 2018, 133, 107-130, doi:10.1016/j.addr.2018.08.012.

47. Pushpakom, S.; Iorio, F.; Eyers, P.A.; Escott, K.J.; Hopper, S.; Wells, A.; Doig, A.; Guilliams, T.; Latimer, J.; McNamee, C.; et al. Drug repurposing: progress, challenges and recommendations. Nat. Rev. Drug Discov. 2019, 18, 41-58, doi:10.1038/nrd.2018.168.

48. Tyagi, A.; Kapoor, P.; Kumar, R.; Chaudhary, K.; Gautam, A.; Raghava, G.P.S. In silico models for designing and discovering novel anticancer peptides. Sci. Rep. 2013, 3, 1-8, doi:10.1038/srep02984.

49. Gautam, A.; Chaudhary, K.; Kumar, R.; Sharma, A.; Kapoor, P.; Tyagi, A.; Raghava, G.P.S. In silico approaches for designing highly effective cell penetrating peptides. J. Transl. Med. 2013, 11, 74, doi:10.1186/1479-5876-11-74.

50. Gupta, S.; Kapoor, P.; Chaudhary, K.; Gautam, A.; Kumar, R.; Raghava, G.P.S. In Silico Approach for Predicting Toxicity of Peptides and Proteins. PLoS One 2013, 8, e73957, doi:10.1371/journal.pone.0073957.

51. Chaudhary, K.; Kumar, R.; Singh, S.; Tuknait, A.; Gautam, A.; Mathur, D.; Anand, P.; Varshney, G.C.; Raghava, G.P.S. A Web Server and Mobile App for Computing Hemolytic Potency of Peptides. Sci. Rep. 2016, 6, 22843, doi:10.1038/srep22843.

Xu, J.; Li, F.; Leier, A.; Xiang, D.; Shen, H.-H.; Marquez Lago, T.T.; Li, J.; Yu, D.-J.; Song, J. Comprehensive assessment of machine learning-based methods for predicting antimicrobial peptides. Brief. Bioinform. 2021, 22, 1-22, doi:10.1093/bib/bbab083.

53. Thomsen, M.C.F.; Nielsen, M. Seq2Logo: A method for construction and visualization of amino acid binding motifs and sequence profiles including sequence weighting, pseudo counts and two-sided representation of amino acid enrichment and depletion. Nucleic Acids Res. 2012, 40, 281-287, doi:10.1093/nar/gks469.

54. Zahoránszky-Kohalmi, G.; Bologa, C.G.; Oprea, T.I. Impact of similarity threshold on the topology of molecular similarity networks and clustering outcomes. J. Cheminform. 2016, 8, 1-17, doi:10.1186/s13321-016-0127-5.

55. Chicco, D.; Tötsch, N.; Jurman, G. The Matthews correlation coefficient (MCC) is more reliable than balanced accuracy, bookmaker informedness, and markedness in two-class confusion matrix evaluation. BioData Min. 2021, 14, 13, doi:10.1186/s13040-021-00244-z.

56. Jobin, M.-L.; Blanchet, M.; Henry, S.; Chaignepain, S.; Manigand, C.; Castano, S.; Lecomte, S.; Burlina, F.; Sagan, S.; Alves, I.D. The role of tryptophans on the cellular uptake and membrane interaction of arginine-rich cell penetrating peptides. Biochim. Biophys. Acta - Biomembr. 2015, 1848, 593-602, doi:10.1016/j.bbamem.2014.11.013.

57. Chu, H.L.; Yip, B.S.; Chen, K.H.; Yu, H.Y.; Chih, Y.H.; Cheng, H.T.; Chou, Y.T.; Cheng, J.W. Novel antimicrobial peptides with high anticancer activity and selectivity. PLoS One 2015, 10, 1-14, doi:10.1371/journal.pone.0126390.

58. Ellerby, H.M.; Arap, W.; Ellerby, L.M.; Kain, R.; Andrusiak, R.; Rio, G. Del; Krajewski, S.; Lombardo, C.R.; Rao, R.; Ruoslahti, E.; et al. Anti-cancer activity of targeted pro-apoptotic peptides. Nat. Med. 1999, 5, 1032-1038, doi:10.1038/12469.

59. Ruoslahti, E.; Pasqualini, R. Tumor Homing molecules, conjugates derived therefrom, and methods of using same 1998.

60. Bayse, C.A.; Pollard, D.B. Conformation dynamics of cyclic disulfides and selenosulfides in CXXC(U) (X=Gly, Ala) tetrapeptide redox motifs. J. Pept. Sci. 2019, 25, 16-22, doi:10.1002/psc.3160.

61. Lee, S.; Kim, S.M.; Lee, R.T. Thioredoxin and thioredoxin target proteins: From molecular mechanisms to functional significance. Antioxidants Redox Signal. 2013, 18, 1165-1207, doi:10.1089/ars.2011.4322.

62. Ohta, T.; Hashida, Y.; Yamashita, F.; Hashida, M. Sustained Release of Mitomycin C from Its Conjugate with Single-Walled Carbon Nanotubes Associated by Pegylated Peptide. Biol. Pharm. Bull. 2016, 39, 1687-1693, doi:10.1248/bpb.b16-00434.

63. Bailey, T.L. DREME: Motif discovery in transcription factor ChIP-seq data. Bioinformatics 2011, 27, 1653-1659, doi:10.1093/bioinformatics/btr261.

64. Heinz, S.; Benner, C.; Spann, N.; Bertolino, E.; Lin, Y.C.; Laslo, P.; Cheng, J.X.; Murre, C.; Singh, H.; Glass, C.K. Simple Combinations of Lineage-Determining Transcription Factors Prime cis-Regulatory Elements Required for Macrophage and B Cell Identities. Mol. Cell 2010, 38, 576-589, doi:10.1016/j.molcel.2010.05.004.

65. Bailey, T.L.; Boden, M.; Buske, F.A.; Frith, M.; Grant, C.E.; Clementi, L.; Ren, J.; Li, W.W.; Noble, W.S. MEME Suite: Tools for 
motif discovery and searching. Nucleic Acids Res. 2009, 37, 202-208, doi:10.1093/nar/gkp335.

66. Asai, T.; Nagatsuka, M.; Kuromi, K.; Yamakawa, S.; Kurohane, K.; Ogino, K.; Tanaka, M.; Taki, T.; Oku, N. Suppression of tumor growth by novel peptides homing to tumor-derived new blood vessels. FEBS Lett. 2002, 510, 206-210, doi:10.1016/S0014-5793(01)03265-3.

67. Oku, N.; Asai, T.; Watanabe, K.; Kuromi, K.; Nagatsuka, M.; Kurohane, K.; Kikkawa, H.; Ogino, K.; Tanaka, M.; Ishikawa, D.; et al. Anti-neovascular therapy using novel peptides homing to angiogenic vessels. Oncogene 2002, 21, 2662-2669, doi:10.1038/sj.onc.1205347.

68. Jennings, B.H.; Pickles, L.M.; Wainwright, S.M.; Roe, S.M.; Pearl, L.H.; Ish-Horowicz, D. Molecular Recognition of Transcriptional Repressor Motifs by the WD Domain of the Groucho/TLE Corepressor. Mol. Cell 2006, 22, 645-655, doi:10.1016/j.molcel.2006.04.024.

69. Castelletto, V.; Edwards-Gayle, C.J.C.; Hamley, I.W.; Pelin, J.N.B.D.; Alves, W.A.; Aguilar, A.M.; Seitsonen, J.; Ruokolainen, J. Self-assembly of a catalytically active lipopeptide and its incorporation into cubosomes. ACS Appl. Bio Mater. 2019, 2, 36393647, doi:10.1021/acsabm.9b00489.

70. Benjdia, A.; Berteau, O. Radical SAM Enzymes and Ribosomally-Synthesized and Post-translationally Modified Peptides: A Growing Importance in the Microbiomes. Front. Chem. 2021, 9, 1-16, doi:10.3389/fchem.2021.678068.

71. Yu, F.-H.; Huang, K.-J.; Wang, C.-T. C-Terminal HIV-1 Transframe p6* Tetrapeptide Blocks Enhanced Gag Cleavage Incurred by Leucine Zipper Replacement of a Deleted p6* Domain. J. Virol. 2017, 91, 1-14, doi:10.1128/jvi.00103-17.

72. Kanehisa, M.; Goto, S.; Kawashima, S.; Nakaya, A. Thed KEGG databases at GenomeNet. Nucleic Acids Res. 2002, 30, 42-46, doi:10.1093/nar/30.1.42.

73. Ohlsson, B. Gonadotropin-releasing hormone and its role in the enteric nervous system. Front. Endocrinol. (Lausanne). 2017, 8, 1-7, doi:10.3389/fendo.2017.00110.

74. Spindel, E.R. Chapter 46 - Bombesin Peptides. In Handbook of Biologically Active Peptides; Kastin, A.J., Ed.; Academic Press: Boston, 2013; pp. 326-330.

75. Guo, M.; Qu, X.; Qin, X.Q. Bombesin-like peptides and their receptors: Recent findings in pharmacology and physiology. Curr. Opin. Endocrinol. Diabetes Obes. 2015, 22, 3-8, doi:10.1097/MED.0000000000000126. 